\title{
MIXED-DIMENSIONAL GEOMETRIC MULTIGRID METHODS FOR SINGLE-PHASE FLOW IN FRACTURED POROUS MEDIA*
}

\author{
ANDRÉS ARRARÁS ${ }^{\dagger}$, FRANCISCO J. GASPAR ${ }^{\ddagger}$, LAURA PORTERO $^{\dagger}$, AND \\ CARMEN RODRIGO§
}

\begin{abstract}
This paper deals with the efficient numerical solution of single-phase flow problems in fractured porous media. A monolithic multigrid method is proposed for solving two-dimensional arbitrary fracture networks with vertical and/or horizontal possibly intersecting fractures. The key point is to combine two-dimensional multigrid components (smoother and intergrid transfer operators) in the porous matrix with their one-dimensional counterparts within the fractures, giving rise to a mixed-dimensional geometric multigrid method. This combination seems to be optimal since it provides an algorithm whose convergence matches the multigrid convergence factor for solving the Darcy problem. Several numerical experiments are presented to demonstrate the robustness of the monolithic mixed-dimensional multigrid method with respect to the permeability of the fractures, the grid size, and the number of fractures in the network.
\end{abstract}

Key words. fracture network, fractured porous media, monolithic multigrid method, singlephase flow, Vanka smoother

AMS subject classifications. $65 \mathrm{~F} 10,65 \mathrm{~N} 22,65 \mathrm{~N} 55,76 \mathrm{~S} 05$

DOI. $10.1137 / 18 \mathrm{M} 1224751$

1. Introduction. The numerical simulation of subsurface flow through fractured porous media is a challenging task which is getting increasing attention in recent years. The essential role played by fractures in different applications - ranging from petroleum extraction to long-term $\mathrm{CO}_{2}$ and nuclear waste storage - demands the design of efficient discretization methods for solving the corresponding flow models. Depending on the spatial scale under consideration, fractures can be incorporated into such models in essentially two ways. At small scales, when specific locations of microfractures are difficult to determine, the so-called dual-porosity models $[11,12]$ are used. In this case, the network of fractures and the bulk or porous matrix are two interacting continua related by a transfer function. On the other hand, at large scales, geological discontinuities represented by localized networks of faults and macrofractures require the use of discrete fracture models $[29,52]$. In these models, fractures can behave either as preferential flow paths or as geological barriers, depending on the permeability contrast between the porous matrix and the fractures themselves.

*Submitted to the journal's Computational Methods in Science and Engineering section November 5, 2018; accepted for publication (in revised form) July 22, 2019; published electronically October $15,2019$.

https://doi.org/10.1137/18M1224751

Funding: The work of the first and fourth authors was supported by Spanish project PGC2018099536-A-I00 (MCIU/AEI/FEDER, UE). The work of the second author was supported by the European Union's Horizon 2020 research and innovation program under the Marie Sklodowska-Curie grant agreement 705402 , POROSOS. The work of the third author was partially supported by the Spanish project FEDER/MCYT MTM2016-75139-R. The work of the fourth author was supported by the DGA (Grupo de referencia APEDIF, ref. E24 17R).

${ }^{\dagger}$ Departamento de Estadística, Informática y Matemáticas, Universidad Pública de Navarra, Edificio de Las Encinas, Campus de Arrosadía, 31006 Pamplona, Spain (andres.arraras@unavarra.es, laura.portero@unavarra.es).

${ }^{\ddagger}$ CWI, Centrum Wiskunde and Informatica, 1098 XG Amsterdam, The Netherlands (gaspar@ cwi.nl).

§IUMA, Departamento de Matemática Aplicada, Universidad de Zaragoza, 50009 Zaragoza, Spain (carmenr@unizar.es). 
Henceforth, we shall consider this latter approach. Discrete fracture models typically require fine meshing of the fracture domain to guarantee accurate approximations. Provided that the fracture aperture is small as compared to the characteristic length of the flow domain, this fact can yield computationally expensive discretizations. To avoid such limitations and based on geometrical model reduction techniques, fractures are represented as $(n-1)$-dimensional interfaces immersed into an $n$-dimensional porous matrix. The resulting model is called a mixed-dimensional $[44,59]$ or reduced $[30,69]$ model. In this framework, flow can be described by several models within the fractures and in the porous matrix. In $[6,8,16,23,30,52]$, incompressible single-phase Darcy flow is considered in both domains. Extensions to two-phase flow can be found, e.g., in [35, 39]. Alternatively, models that consider high-velocity flows within the fractures include Darcy-Brinkman [19, 47], Forchheimer $[33,45]$, and Reynolds lubrication [38] equations.

In this paper, we focus on the single-phase Darcy-Darcy coupling between the fractures and the porous matrix. The governing equations comprise a system of mixed-dimensional partial differential equations [15] defined on the $n$-dimensional porous matrix, $(n-1)$-dimensional fractures, and $(n-2)$-dimensional intersections between fractures. This problem has been extensively addressed in the literature by means of different discretization techniques. Raviart-Thomas mixed finite element schemes are studied, e.g., in $[6,52]$ for the case of conforming meshes on the fracture interfaces. Their extension to nonmatching grids is discussed in [32] and, suitably combined with mortar methods, in [16]. In both cases, however, the geometry of the fractures is respected by the meshes. Alternative strategies that permit meshing the entire domain independently of the fractures include the so-called embedded discrete fracture-matrix methods $[41,56]$ and the extended finite element methods (XFEM) $[23,29]$ (and references therein). In the former case, the fracture grid points are located at the intersection between the background mesh and the fracture itself. In the latter, they can be arbitrarily placed with respect to the matrix mesh. In addition, further discretization schemes have been proposed for handling general elements and distorted grids, namely, mimetic finite difference methods [10, 31], discontinuous Galerkin methods [9], virtual element methods [14, 34], hybrid high-order methods [21], or multipoint flux approximation methods [5,66]. Here, we consider a rectangular grid that is conforming at the fractures and a discretization based on mixed finite element methods which is equivalent to a finite volume scheme after the application of appropriate quadrature rules.

Although a lot of effort has been put into developing numerical schemes for the discretization of fracture models, efficient solvers for the resulting linear systems have not been so deeply investigated. A first step in this direction is the design of linear solvers for discrete fracture network models, which consider the solution of the flow equations only within the fracture network without taking into account the coupling with the surrounding porous matrix. The discussion of different solution approaches in this context can be found in [24]. Some relevant works related to the development of solvers for the discretization of the mixed-dimensional model considered here include iterative strategies in a domain decomposition framework [23, 52] (see also [29] for a discussion on linear solvers and [43] for an extension to time-dependent problems), physics-based preconditioners [67], or iterative multiscale methods [41, 77]. In this context, the aim of this paper is to develop a monolithic geometric multigrid method for solving mixed-dimensional Darcy problems on fractured porous media. To the best of our knowledge, this is the first time that a similar approach is proposed in the literature. For the ease of presentation, we shall assume a distribution of horizontal 
and vertical fractures that can intersect with each other in virtually any way. This fracture configuration can be efficiently discretized by means of conforming mixed methods based on Raviart-Thomas elements. Further applying suitable quadrature rules, we can derive finite volume schemes that extend the ideas proposed in [65] for unfractured domains. This scheme yields a linear system with a saddle-point structure to solve. This is an important property of the system that influences the design of the multigrid method described below. Other discretization approaches, such as mixed hybrid finite element methods, would give rise to a symmetric positive definite (SPD) matrix, which could be efficiently solved by using, e.g., a preconditioned conjugate gradient method. In that same framework, the velocities could be eliminated, thus resulting in a linear system with only pressure unknowns involving a Schur complement matrix that would also be SPD. The idea of applying multigrid methods could be considered in such a context as well, providing very efficient solution strategies for the SPD system, either as stand-alone solvers or as preconditioners for the conjugate gradient method. In addition to the monolithic multigrid solver, we also introduce here a novel representation of the network of fractures based on graph theory.

It is well known that multigrid methods $[17,40,73,78,80]$ are among the fastest numerical techniques for solving the large systems of equations arising from the discretization of partial differential equations. They have shown optimal complexity in solving many problems in different areas of application [78]. However, it is the first time that multigrid is applied for solving a mixed-dimensional flow problem in fractured porous media. These algorithms strongly depend on the appropriate choice of their components, mainly the intergrid transfer operators and the smoother. In this work, a mixed-dimensional geometric multigrid method is proposed to deal with the complex mixed-dimensional problem at once. In a two-dimensional setting, the proposed multigrid solver suitably combines two-dimensional smoother and intergrid transfer operators in the porous matrix with their one-dimensional counterparts within the fracture network. Due to the saddle-point character of the whole resulting system, we choose a multiplicative Schwarz smoother, which has been proved to be efficient for different problems in porous media. The resulting mixed-dimensional monolithic multigrid method shows robustness with respect to the mesh size, the permeability of the fractures, and the number of fractures in the network.

The rest of the paper is organized as follows. Section 2 describes the mixeddimensional model problem and the spatial discretization considered. In particular, we first focus on the case of a single fracture, and then we address the general case of multiple intersecting fractures. Section 3 introduces a mixed-dimensional monolithic multigrid method that combines two-dimensional components in the porous matrix with one-dimensional components in the fractures. Section 4 shows several numerical experiments considering various fracture configurations and permeability distributions that confirm the robustness of the proposed solver. Finally, section 5 contains some concluding remarks.

2. Model problem and discretization. In this section, we introduce the system of equations modeling single-phase Darcy flow in a fractured porous medium. For the ease of presentation, the model is first derived for the case of a single fracture. The weak formulation and its MFE discretization are then obtained. Next, we address the case of intersecting fractures and emphasize the key points of this extended model. In both cases, the resulting algebraic system for the approximation scheme is provided.

2.1. A single fracture model problem. Let $\Omega \subset \mathbb{R}^{2}$ be an open, bounded, and convex polygonal domain whose boundary is denoted by $\Gamma=\partial \Omega$. We consider a 

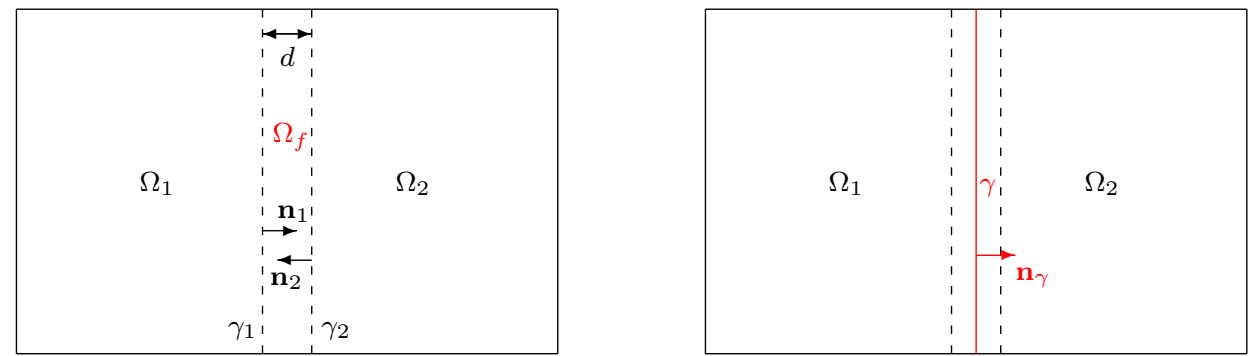

FIG. 1. Schematic representation of the original domain (left) and the reduced domain (right).

single-phase, incompressible flow in $\Omega$, governed by Darcy's law in combination with the mass conservation equation, i.e.,

$$
\begin{aligned}
\mathbf{u} & =-\mathbf{K} \nabla p & & \text { in } \Omega, \\
\nabla \cdot \mathbf{u} & =q & & \text { in } \Omega, \\
p & =0 & & \text { on } \Gamma .
\end{aligned}
$$

Here, $p$ denotes the pressure, $\mathbf{u}$ is the Darcy velocity, $\mathbf{K} \in \mathbb{R}^{2 \times 2}$ is the permeability tensor, and $q$ is the source/sink term. We suppose that $\mathbf{K}$ is a diagonal tensor whose entries are strictly positive and bounded in $\Omega$. Homogeneous Dirichlet boundary conditions have been considered for simplicity, but other types of boundary data can also be handled.

To begin with, we suppose that the porous matrix (or bulk) $\Omega$ contains a subset $\Omega_{f}$ representing a single fracture, which divides the flow domain into two disjoint, connected subdomains $\Omega_{1}$ and $\Omega_{2}$, i.e.,

$$
\Omega \backslash \bar{\Omega}_{f}=\Omega_{1} \cup \Omega_{2}, \quad \Omega_{1} \cap \Omega_{2}=\emptyset .
$$

We further denote $\Gamma_{i}=\partial \Omega_{i} \cap \Gamma$ for $i=1,2, f$ and $\gamma_{i}=\partial \Omega_{i} \cap \partial \Omega_{f} \cap \Omega$ for $i=1,2$. The unit vector normal to $\gamma_{i}$ pointing outward from $\Omega_{i}$ is denoted by $\mathbf{n}_{i}$ for $i=1,2$; see Figure 1 (left).

If we denote by $p_{i}, \mathbf{u}_{i}, \mathbf{K}_{i}$, and $q_{i}$ the restrictions of $p, \mathbf{u}, \mathbf{K}$, and $q$, respectively, to $\Omega_{i}$ for $i=1,2, f$, then (2.1) are equivalent to the following transmission problem for $i=1,2, f$ and $j=1,2$ :

$$
\begin{aligned}
\mathbf{u}_{i} & =-\mathbf{K}_{i} \nabla p_{i} & & \text { in } \Omega_{i}, \\
\nabla \cdot \mathbf{u}_{i} & =q_{i} & & \text { in } \Omega_{i}, \\
p_{j} & =p_{f} & & \text { on } \gamma_{j}, \\
\mathbf{u}_{j} \cdot \mathbf{n}_{j} & =\mathbf{u}_{f} \cdot \mathbf{n}_{j} & & \text { on } \gamma_{j}, \\
p_{i} & =0 & & \text { on } \Gamma_{i} .
\end{aligned}
$$

Note that (2.2c) and (2.2d) provide coupling conditions that guarantee the continuity of the pressure and the normal flux, respectively, across the interfaces between $\Omega_{f}$ and $\Omega_{i}$ for $i=1,2$.

The model provided by $(2.2 \mathrm{a})-(2.2 \mathrm{e})$ is sometimes referred to as an equidimensional model [29] and assumes that both the bulk and the fracture domains share 
the same dimension. As an alternative to this model, we shall define the so-called mixed-dimensional or reduced model, in which the fracture is viewed as a manifold of codimension one (that is, an interface between the bulk subdomains $\Omega_{1}$ and $\Omega_{2}$ ). Based on model reduction techniques, this idea was first proposed in [52] and is commonly used in the framework of fractured porous media [7, 25, 29, 30, 32]. From a numerical viewpoint, the mixed-dimensional approach avoids fine meshing of the fracture domain, thus reducing the computational cost of the overall discretization.

According to [52], there exists a nonself-intersecting one-dimensional manifold $\gamma$ such that the fracture can be expressed as

$$
\Omega_{f}=\left\{\mathbf{x} \in \Omega: \mathbf{x}=\mathbf{s}+\theta \mathbf{n}_{\gamma} \text { for some } \mathbf{s} \in \gamma \text { and }|\theta|<\frac{d(\mathbf{s})}{2}\right\}
$$

where $d(\mathbf{s})>0$ denotes the thickness of the fracture at $\mathbf{s}$ in the normal direction and $\mathbf{n}_{\gamma}$ is the outward unit normal to $\gamma$ with a fixed orientation from $\Omega_{1}$ to $\Omega_{2}$. Note that, with this definition, $\mathbf{n}_{\gamma}=\mathbf{n}_{1}=-\mathbf{n}_{2}$ (see Figure 1). We will assume that the thickness is smaller than the other characteristic dimensions of the fracture.

The key point in this procedure is to collapse the fracture $\Omega_{f}$ into the line $\gamma$ and integrate (2.2a) and (2.2b) for the index $f$ along the fracture thickness. In doing so, we need to split up such equations into their normal and tangential parts. Let us denote the projection operators onto the normal and tangent spaces of $\gamma$ as $\mathbf{P}_{\mathbf{n}}=\mathbf{n}_{\gamma} \mathbf{n}_{\gamma}^{T}$ and $\mathbf{P}_{\boldsymbol{\tau}}=\mathbf{I}-\mathbf{P}_{\mathbf{n}}$, I being the identity tensor. For regular vector- and scalar-valued functions $\mathbf{g}$ and $g$, the tangential divergence and gradient operators on the fracture are defined, respectively, as

$$
\nabla^{\boldsymbol{\tau}} \cdot \mathbf{g}=\mathbf{P}_{\boldsymbol{\tau}}: \nabla \mathbf{g}, \quad \nabla^{\boldsymbol{\tau}} g=\mathbf{P}_{\boldsymbol{\tau}} \nabla g .
$$

Following [30], we assume that the permeability tensor $\mathbf{K}_{f}$ decomposes additively as

$$
\mathbf{K}_{f}=K_{f}^{\mathbf{n}} \mathbf{P}_{\mathbf{n}}+K_{f}^{\tau} \mathbf{P}_{\boldsymbol{\tau}}
$$

where $K_{f}^{\mathbf{n}}$ and $K_{f}^{\tau}$ are defined to be strictly positive and bounded in $\Omega_{f}$.

In this framework, we introduce the so-called reduced variables, namely, the reduced pressure $p_{\gamma}$, the reduced Darcy velocity $\mathbf{u}_{\gamma}$, and the reduced source/sink term $q_{\gamma}$, formally defined as $[25,52]$

$$
p_{\gamma}(\mathbf{s})=\frac{1}{d(\mathbf{s})}\left(p_{f}, 1\right)_{\ell(\mathbf{s})}, \quad \mathbf{u}_{\gamma}(\mathbf{s})=\left(\mathbf{P}_{\tau} \mathbf{u}_{f}, 1\right)_{\ell(\mathbf{s})}, \quad q_{\gamma}(\mathbf{s})=\left(q_{f}, 1\right)_{\ell(\mathbf{s})}
$$

where $\ell(\mathbf{s})=\left(-\frac{d(\mathbf{s})}{2}, \frac{d(\mathbf{s})}{2}\right)$. Hence, we obtain the following interface problem for $i=1,2$ :

$$
\begin{aligned}
\mathbf{u}_{i} & =-\mathbf{K}_{i} \nabla p_{i} & & \text { in } \Omega_{i}, \\
\nabla \cdot \mathbf{u}_{i} & =q_{i} & & \text { in } \Omega_{i}, \\
\mathbf{u}_{\gamma} & =-d K_{f}^{\tau} \nabla^{\boldsymbol{\tau}} p_{\gamma} & & \text { on } \gamma, \\
\nabla^{\tau} \cdot \mathbf{u}_{\gamma} & =q_{\gamma}+\left(\mathbf{u}_{1} \cdot \mathbf{n}_{1}+\mathbf{u}_{2} \cdot \mathbf{n}_{2}\right) & & \text { on } \gamma, \\
\alpha_{\gamma}\left(p_{i}-p_{\gamma}\right) & =\xi \mathbf{u}_{i} \cdot \mathbf{n}_{i}-(1-\xi) \mathbf{u}_{i+1} \cdot \mathbf{n}_{i+1} & & \text { on } \gamma, \\
p_{i} & =0 & & \text { on } \Gamma_{i}, \\
p_{\gamma} & =0 & & \text { on } \partial \gamma,
\end{aligned}
$$


where $\alpha_{\gamma}=2 K_{f}^{\mathbf{n}} / d$ and the index $i$ is supposed to vary in $\mathbb{Z} / 2 \mathbb{Z}$ so that if $i=2$, then $i+1=1$. Following $[8,52], \xi \in(1 / 2,1]$ is a closure parameter related to the pressure cross profile in the fracture. The ratio $K_{f}^{\mathbf{n}} / d$ and the product $K_{f}^{\tau} d$ are sometimes referred to as effective permeabilities in the normal and tangential directions to the fracture, respectively [29].

In the preceding system, (2.5c) represents Darcy's law in the tangential direction of the fracture, while (2.5d) models mass conservation inside the fracture. Remarkably, the additional source term $\mathbf{u}_{1} \cdot \mathbf{n}_{1}+\mathbf{u}_{2} \cdot \mathbf{n}_{2}$ is introduced on $\gamma$ to take into account the contribution of the subdomain flows to the fracture flow. In turn, (2.5e) is obtained by averaging Darcy's law in the normal direction to the fracture and using a quadrature rule with weights $\xi$ and $1-\xi$ for integrating $\mathbf{u}_{f} \cdot \mathbf{n}_{i}$ across the fracture for $i=1,2$. Formally, it can be regarded as a Robin boundary condition for the subdomain $\Omega_{i}$ that involves the pressure in the fracture $p_{\gamma}$ and the normal flux from the neighboring subdomain $\Omega_{i+1}$. It is quite usual to express (2.5e) in terms of average operators for the pressures and normal fluxes, and jump operators for the pressures across the fracture $[23,25]$.

2.2. Weak formulation. In this subsection, we present the weak formulation of the interface problem stated above. To this end, we first introduce the function spaces

$$
\begin{aligned}
& \mathbf{W}=\left\{\mathbf{v}=\left(\mathbf{v}_{1}, \mathbf{v}_{2}, \mathbf{v}_{\gamma}\right) \in H\left(\operatorname{div}, \Omega_{1}\right) \times H\left(\operatorname{div}, \Omega_{2}\right) \times H\left(\operatorname{div}^{\boldsymbol{\tau}}, \gamma\right): \mathbf{v}_{i} \cdot \mathbf{n}_{i} \in L^{2}(\gamma)\right. \\
& \text { for } i=1,2\}, \\
& M=\left\{r=\left(r_{1}, r_{2}, r_{\gamma}\right) \in L^{2}\left(\Omega_{1}\right) \times L^{2}\left(\Omega_{2}\right) \times L^{2}(\gamma)\right\}
\end{aligned}
$$

endowed with the norms [52]

$$
\begin{aligned}
\|\mathbf{v}\|_{\mathbf{W}}^{2}=\sum_{i=1}^{2}\left(\left\|\mathbf{v}_{i}\right\|_{L^{2}\left(\Omega_{i}\right)}^{2}+\left\|\nabla \cdot \mathbf{v}_{i}\right\|_{L^{2}\left(\Omega_{i}\right)}^{2}+\left\|\mathbf{v}_{i} \cdot \mathbf{n}_{i}\right\|_{L^{2}(\gamma)}^{2}\right) & +\left\|\mathbf{v}_{\gamma}\right\|_{L^{2}(\gamma)}^{2}+\left\|\nabla^{\boldsymbol{\tau}} \cdot \mathbf{v}_{\gamma}\right\|_{L^{2}(\gamma)}^{2}, \\
\|r\|_{M}^{2} & =\sum_{i=1}^{2}\left\|r_{i}\right\|_{L^{2}\left(\Omega_{i}\right)}^{2}+\left\|r_{\gamma}\right\|_{L^{2}(\gamma)}^{2} .
\end{aligned}
$$

Here, we use the well-known spaces

$$
\begin{aligned}
& H\left(\operatorname{div}, \Omega_{i}\right)=\left\{\mathbf{v}_{i} \in\left(L^{2}\left(\Omega_{i}\right)\right)^{2}: \nabla \cdot \mathbf{v}_{i} \in L^{2}\left(\Omega_{i}\right)\right\}, \quad i=1,2 \\
& H\left(\operatorname{div}^{\boldsymbol{\tau}}, \gamma\right)=\left\{\mathbf{v}_{\gamma} \in\left(L^{2}(\gamma)\right)^{2}: \nabla^{\boldsymbol{\tau}} \cdot \mathbf{v}_{\gamma} \in L^{2}(\gamma)\right\}
\end{aligned}
$$

and assume that the elements $\mathbf{v}_{\gamma} \in H\left(\operatorname{div}^{\boldsymbol{\tau}}, \gamma\right)$ are aligned with $\gamma$, that is, $\mathbf{v}_{\gamma}=v_{\gamma} \boldsymbol{\tau}$, where $\boldsymbol{\tau}$ denotes the tangent vector to $\gamma$. Note that, in order to take into proper account the Robin boundary condition, we need more regularity in $\mathbf{W}$ than the usual $H($ div, $\cdot)$-regularity commonly used for weak formulations in the context of mixed finite element methods [52]. 
Let $a: \mathbf{W} \times \mathbf{W} \rightarrow \mathbb{R}$ and $b: \mathbf{W} \times M \rightarrow \mathbb{R}$ be the bilinear forms defined by

$$
\begin{aligned}
a(\mathbf{u}, \mathbf{v})= & \sum_{i=1}^{2}\left(\mathbf{K}_{i}^{-1} \mathbf{u}_{i}, \mathbf{v}_{i}\right)_{\Omega_{i}}+\left(\left(d K_{f}^{\boldsymbol{\tau}}\right)^{-1} \mathbf{u}_{\gamma}, \mathbf{v}_{\gamma}\right)_{\gamma} \\
& +\sum_{i=1}^{2}\left(\alpha_{\gamma}^{-1}\left(\xi \mathbf{u}_{i} \cdot \mathbf{n}_{i}-(1-\xi) \mathbf{u}_{i+1} \cdot \mathbf{n}_{i+1}\right), \mathbf{v}_{i} \cdot \mathbf{n}_{i}\right)_{\gamma}, \\
b(\mathbf{u}, r)= & \sum_{i=1}^{2}\left(\nabla \cdot \mathbf{u}_{i}, r_{i}\right)_{\Omega_{i}}+\left(\nabla^{\tau} \cdot \mathbf{u}_{\gamma}, r_{\gamma}\right)_{\gamma}-\left(\mathbf{u}_{1} \cdot \mathbf{n}_{1}+\mathbf{u}_{2} \cdot \mathbf{n}_{2}, r_{\gamma}\right)_{\gamma} .
\end{aligned}
$$

Accordingly, let $L: M \rightarrow \mathbb{R}$ be the linear form associated with the source terms, i.e.,

$$
L(r)=\sum_{i=1}^{2}\left(q_{i}, r_{i}\right)_{\Omega_{i}}+\left(q_{\gamma}, r_{\gamma}\right)_{\gamma} .
$$

In this framework, the weak formulation of the interface problem (2.5) reads as follows: Find $(\mathbf{u}, p) \in \mathbf{W} \times M$ such that

$$
\begin{aligned}
a(\mathbf{u}, \mathbf{v})-b(\mathbf{v}, p) & =0 & & \forall \mathbf{v} \in \mathbf{W}, \\
b(\mathbf{u}, r) & =L(r) & & \forall r \in M .
\end{aligned}
$$

The existence and uniqueness of solution to this problem is proved in [52] for the case $\xi>1 / 2$, assuming that the permeabilities in both subdomains and the coefficients $K_{f}^{\mathbf{n}} / d$ and $K_{f}^{\tau} d$ are bounded by positive constants.

2.3. Mixed finite element approximation. Let us assume that the subdomains $\Omega_{i}$ admit rectangular partitions $\mathcal{T}_{i}^{h}$, for $i=1,2$, that match at the interface $\gamma$. Such meshes $\mathcal{T}_{i}^{h}$ induce a unique partition on $\gamma$ denoted by $\mathcal{T}_{\gamma}^{h}$.

Let $\mathbf{W}_{i}^{h} \times M_{i}^{h}$ be the lowest order Raviart-Thomas mixed finite element spaces defined on $\mathcal{T}_{i}^{h}$, for $i=1,2, \gamma$, and let us introduce the global spaces

$$
\mathbf{W}^{h}=\bigoplus_{i=1,2, \gamma} \mathbf{W}_{i}^{h}, \quad M^{h}=\bigoplus_{i=1,2, \gamma} M_{i}^{h} .
$$

Following [13, 65], we will use numerical quadrature rules for evaluating some of the integrals in (2.6). More specifically, based on the expression for $a(\mathbf{u}, \mathbf{v})$ defined above, we set the following discrete bilinear form:

$$
\begin{aligned}
a_{h}(\mathbf{u}, \mathbf{v})= & \sum_{i=1}^{2}\left(\mathbf{K}_{i}^{-1} \mathbf{u}_{i}, \mathbf{v}_{i}\right)_{\Omega_{i}, \mathbf{T M}}+\left(\left(d K_{f}^{\boldsymbol{\tau}}\right)^{-1} \mathbf{u}_{\gamma}, \mathbf{v}_{\gamma}\right)_{\gamma, \mathbf{T}} \\
& +\sum_{i=1}^{2}\left(\alpha_{\gamma}^{-1}\left(\xi \mathbf{u}_{i} \cdot \mathbf{n}_{i}-(1-\xi) \mathbf{u}_{i+1} \cdot \mathbf{n}_{i+1}\right), \mathbf{v}_{i} \cdot \mathbf{n}_{i}\right)_{\gamma}
\end{aligned}
$$

where $(\cdot, \cdot)_{\gamma, \mathbf{T}}$ denotes the application of the trapezoidal rule for computing the innerproduct integral over $\gamma$ and $(\cdot, \cdot)_{\Omega_{i}}$, TM is defined, for any vector-valued functions $\mathbf{f}, \mathbf{g} \in \mathbb{R}^{2}$, as $[13,65]$

$$
(\mathbf{f}, \mathbf{g})_{\Omega_{i}, \mathbf{T M}}=\left(f_{1}, g_{1}\right)_{\Omega_{i}, \mathbf{T} \times \mathbf{M}}+\left(f_{2}, g_{2}\right)_{\Omega_{i}, \mathbf{M} \times \mathbf{T}} .
$$


In this case, the integral of the $i$ th component of the vectors, for $i=1,2$, is computed by using the trapezoidal rule in the $i$ th direction and the midpoint rule in the other direction. On the other hand, the discrete counterpart to $L(r)$ is given by

$$
L_{h}(r)=\sum_{i=1}^{2}\left(q_{i}, r_{i}\right)_{\Omega_{i}, \mathbf{M}}+\left(q_{\gamma}, r_{\gamma}\right)_{\gamma, \mathbf{M}},
$$

where $(\cdot, \cdot)_{G, \mathbf{M}}$ means the application of the midpoint rule for computing the corresponding inner-product integral over $G$. In this context, the mixed finite element approximation to (2.6) may be written as follows: Find $\left(\mathbf{u}_{h}, p_{h}\right) \in \mathbf{W}^{h} \times M^{h}$ such that

$$
\begin{aligned}
a_{h}\left(\mathbf{u}_{h}, \mathbf{v}_{h}\right)-b\left(\mathbf{v}_{h}, p_{h}\right) & =0 & & \forall \mathbf{v}_{h} \in \mathbf{W}^{h}, \\
b\left(\mathbf{u}_{h}, r_{h}\right) & =L_{h}\left(r_{h}\right) & & \forall r_{h} \in M^{h} .
\end{aligned}
$$

Note that the definition of the global spaces $\mathbf{W}^{h}$ and $M^{h}$ implies $\mathbf{u}_{h}=\left(\mathbf{u}_{1}^{h}, \mathbf{u}_{2}^{h}, \mathbf{u}_{\gamma}^{h}\right)$ and $p_{h}=\left(p_{1}^{h}, p_{2}^{h}, p_{\gamma}^{h}\right)$. Following [65], it can be proved that this method is closely related to the so-called two-point flux approximation method [26].

2.4. Algebraic linear system. Let $\left\{\mathbf{v}_{k, i}\right\}_{i=1}^{E_{k}}$ and $\left\{r_{k, i}\right\}_{i=1}^{C_{k}}$ denote the basis functions of $\mathbf{W}_{k}^{h}$ and $M_{k}^{h}$, respectively, for $k=1,2, \gamma$. Here, $E_{k}$ and $C_{k}$ stand for the number of edges and cells in $\mathcal{T}_{k}^{h}$, respectively. Thus, the unknowns in (2.7) can be expressed as

$$
\mathbf{u}_{k}^{h}=\sum_{i=1}^{E_{k}} U_{k, i} \mathbf{v}_{k, i}, \quad p_{k}^{h}=\sum_{i=1}^{C_{k}} P_{k, i} r_{k, i},
$$

for $k=1,2, \gamma$. If we define the vectors $U_{k} \in \mathbb{R}^{E_{k}}$ and $P_{k} \in \mathbb{R}^{C_{k}}$ with components $U_{k, i}$ and $P_{k, i}$, respectively, for $k=1,2, \gamma$, then the algebraic linear system stemming from (2.7) is a saddle-point problem of the form

$$
\left[\begin{array}{cccccc}
A_{1} & D^{T} & 0 & B_{1}^{T} & 0 & F_{1}^{T} \\
D & A_{2} & 0 & 0 & B_{2}^{T} & F_{2}^{T} \\
0 & 0 & A_{\gamma} & 0 & 0 & B_{\gamma}^{T} \\
B_{1} & 0 & 0 & 0 & 0 & 0 \\
0 & B_{2} & 0 & 0 & 0 & 0 \\
F_{1} & F_{2} & B_{\gamma} & 0 & 0 & 0
\end{array}\right]\left[\begin{array}{c}
U_{1} \\
U_{2} \\
U_{\gamma} \\
P_{1} \\
P_{2} \\
P_{\gamma}
\end{array}\right]=\left[\begin{array}{c}
0 \\
0 \\
0 \\
Q_{1} \\
Q_{2} \\
Q_{\gamma}
\end{array}\right] .
$$

In particular, the entries of the matrices $A_{k} \in \mathbb{R}^{E_{k} \times E_{k}}, B_{k} \in \mathbb{R}^{C_{k} \times E_{k}}$, and $F_{k} \in$ $\mathbb{R}^{C_{\gamma} \times E_{k}}$, for $k=1,2, D \in \mathbb{R}^{E_{2} \times E_{1}}, A_{\gamma} \in \mathbb{R}^{E_{\gamma} \times E_{\gamma}}$, and $B_{\gamma} \in \mathbb{R}^{C_{\gamma} \times E_{\gamma}}$, are

$$
\begin{array}{rlr}
{\left[A_{k}\right]_{i, j}} & =\left(\mathbf{K}_{k}^{-1} \mathbf{v}_{k, j}, \mathbf{v}_{k, i}\right)_{\Omega_{k}, \mathbf{T M}}+\alpha_{\gamma}^{-1}\left(\xi \mathbf{v}_{k, j} \cdot \mathbf{n}_{k}, \mathbf{v}_{k, i} \cdot \mathbf{n}_{k}\right)_{\gamma}, & k=1,2, \\
{\left[B_{k}\right]_{i, j}} & =-\left(r_{k, i}, \nabla \cdot \mathbf{v}_{k, j}\right)_{\Omega_{k}}, & k=1,2, \\
{\left[F_{k}\right]_{i, j}} & =\left(r_{\gamma, i}, \mathbf{v}_{k, j} \cdot \mathbf{n}_{k}\right)_{\gamma}, & \\
{[D]_{i, j}} & =\alpha_{\gamma}^{-1}\left((\xi-1) \mathbf{v}_{2, i} \cdot \mathbf{n}_{2}, \mathbf{v}_{1, j} \cdot \mathbf{n}_{1}\right)_{\gamma}, & \\
{\left[A_{\gamma}\right]_{i, j}} & =\left(\left(d K_{f}^{\boldsymbol{\tau}}\right)^{-1} \mathbf{v}_{\gamma, j}, \mathbf{v}_{\gamma, i}\right)_{\gamma, \mathbf{T}}, & \\
{\left[B_{\gamma}\right]_{i, j}} & =-\left(r_{\gamma, i}, \nabla^{\tau} \cdot \mathbf{v}_{\gamma, j}\right)_{\gamma}, &
\end{array}
$$


where []$_{i, j}$ indicates the $(i, j)$ th element of the matrix. Note that the use of the quadrature rules $(\cdot, \cdot)_{\Omega_{k}, \mathbf{T M}}$ and $(\cdot, \cdot)_{\gamma, \mathbf{T}}$ diagonalizes the matrices $A_{1}, A_{2}$, and $A_{\gamma}$. In turn, the components of the vectors $Q_{k} \in \mathbb{R}^{C_{k}}$, for $k=1,2, \gamma$, are given by

$$
\begin{aligned}
{\left[Q_{k}\right]_{j} } & =-\left(q_{k}, r_{k, j}\right)_{\Omega_{k}, \mathbf{M}}, \quad k=1,2, \\
{\left[Q_{\gamma}\right]_{j} } & =-\left(q_{\gamma}, r_{\gamma, j}\right)_{\gamma, \mathbf{M}},
\end{aligned}
$$

where $[\cdot]_{j}$ denotes the $j$ th component of the vector.

The next subsection is devoted to the general case of multiple intersecting fractures. With the aim of achieving a unified notation, we shall group the unknowns corresponding to the two-dimensional bulk subdomains into the vectors $U^{2}=\left[U_{1}, U_{2}\right]^{T}$ and $P^{2}=\left[P_{1}, P_{2}\right]^{T}$ and those associated with the one-dimensional fractures into $U^{1}=U_{\gamma}$ and $P^{1}=P_{\gamma}$. In these cases, the superscripts stand for the corresponding dimensions. Using such notations, the preceding system can be rewritten as

$$
\left[\begin{array}{cccc}
A_{2,2} & 0 & B_{2,2}^{T} & F_{2,1}^{T} \\
0 & A_{1,1} & 0 & B_{1,1}^{T} \\
B_{2,2} & 0 & 0 & 0 \\
F_{2,1} & B_{1,1} & 0 & 0
\end{array}\right]\left[\begin{array}{c}
U^{2} \\
U^{1} \\
P^{2} \\
P^{1}
\end{array}\right]=\left[\begin{array}{c}
0 \\
0 \\
Q^{2} \\
Q^{1}
\end{array}\right]
$$

where, accordingly, $Q^{2}=\left[Q_{1}, Q_{2}\right]^{T}$ and $Q^{1}=Q_{\gamma}$.

Remark 1 . In the case of considering nonhomogeneous Dirichlet boundary conditions at any part of the boundary of the porous matrix and/or the fracture, the right-hand side of (2.6a) and (2.7a) would be different from zero. As a consequence, some of the upper components of the independent term in systems (2.8) and (2.9) would also be nonzero. In turn, assuming Neumann boundary conditions at any part of the boundary of the porous matrix and/or the fracture would affect not only the systems (2.8) and (2.9) but also the definition of the function spaces $\mathbf{W}$ and $\mathbf{W}_{h}$.

2.5. The case of multiple intersecting fractures. Let us now consider problem (2.1) posed on a geological domain subdivided into $m$ subdomains $\Omega_{i}$ for $i \in \mathcal{I}_{2}=$ $\{1,2, \ldots, m\}$, naturally separated by a collection of fractures $\Omega_{i, j}$ for $(i, j) \in \mathcal{I}_{1}$. Here and henceforth, $\mathcal{I}_{1}$ is a set of indices $(i, j)$, with $i, j \in \mathcal{I}_{2}$, and $i<j$, that permits us to label the fractures. In particular, $\Omega_{i, j}$ denotes a fracture that is adjacent to subdomains $\Omega_{i}$ and $\Omega_{j}$. In this framework, it holds that

$$
\Omega \backslash\left(\bigcup_{(i, j) \in \mathcal{I}_{1}} \bar{\Omega}_{i, j}\right)=\bigcup_{i \in \mathcal{I}_{2}} \Omega_{i}
$$

with $\Omega_{i} \cap \Omega_{j}=\emptyset$ for $i \neq j$.

Following the ideas of the preceding subsection, let us suppose that there exist certain nonself-intersecting one-dimensional manifolds $\gamma_{i, j}$ such that the fractures can be defined as

$$
\Omega_{i, j}=\left\{\mathbf{x} \in \Omega: \mathbf{x}=\mathbf{s}+\theta \mathbf{n}_{i, j} \text { for some } \mathbf{s} \in \gamma_{i, j} \text { and }|\theta|<\frac{d_{i, j}(\mathbf{s})}{2}\right\},
$$

where $d_{i, j}(\mathbf{s})$ denotes the thickness of the fracture $\Omega_{i, j}$ at $\mathbf{s}$ in the normal direction and $\mathbf{n}_{i, j}$ is the outward unit normal to $\gamma_{i, j}$ oriented from $\Omega_{i}$ to $\Omega_{j}$ for $(i, j) \in \mathcal{I}_{1}$. Let us denote by $\left\{\sigma_{i, j, k}\right\}_{(i, j, k) \in \mathcal{I}_{0}^{T}}$ the $T$-shaped intersections of three fractures (i.e., 

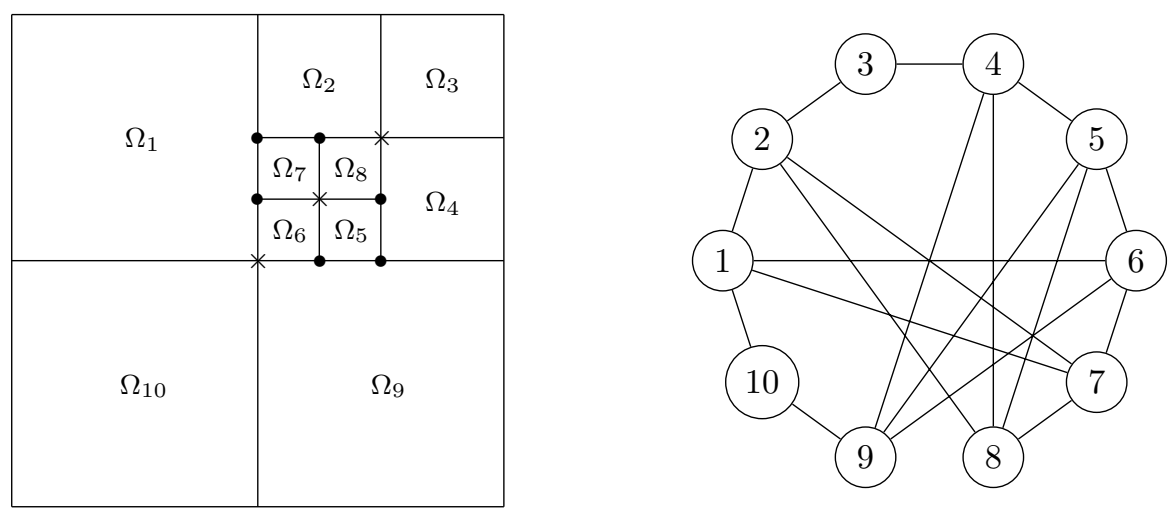

FIG. 2. Schematic representation of the reduced domain (left) and the associated graph (right).

$\left.\sigma_{i, j, k}=\bar{\gamma}_{i, j} \cap \bar{\gamma}_{j, k} \cap \bar{\gamma}_{i, k}\right)$ and by $\left\{\sigma_{i, j, k, l}\right\}_{(i, j, k, l) \in \mathcal{I}_{0}^{X}}$ the $X$-shaped intersections of four fractures (for instance, $\sigma_{i, j, k, l}=\bar{\gamma}_{i, j} \cap \bar{\gamma}_{j, k} \cap \bar{\gamma}_{k, l} \cap \bar{\gamma}_{i, l}$ ).

In the following, we present an example to illustrate the used notations for a flow domain containing both horizontal and vertical fractures that may intersect. Figure 2 (left) shows the schematic representation of a domain that contains $m=10$ subdomains and 18 fractures. This geometry will be later considered as a benchmark problem in the section devoted to the numerical experiments (cf. subsection 4.3).

In general, given $i=1,2, \ldots, m-1$, let us define the set $\mathcal{N}_{i}$ that contains the indices of the subdomains $\Omega_{j}$, with $j>i$, that are adjacent to $\Omega_{i}$. Specifically, in this example, we have

$$
\begin{array}{lll}
\mathcal{N}_{1}=\{2,6,7,10\}, & \mathcal{N}_{4}=\{5,8,9\}, & \mathcal{N}_{7}=\{8\}, \\
\mathcal{N}_{2}=\{3,7,8\}, & \mathcal{N}_{5}=\{6,8,9\}, & \mathcal{N}_{8}=\emptyset, \\
\mathcal{N}_{3}=\{4\}, & \mathcal{N}_{6}=\{7,9\}, & \mathcal{N}_{9}=\{10\} .
\end{array}
$$

Then the set of indices $\mathcal{I}_{1}$ denoting the one-dimensional collapsed fractures may be defined as

$$
\mathcal{I}_{1}=\left\{(i, j) \in \mathbb{N}^{2}: i \in\{1,2, \ldots, m-1\}, j \in \mathcal{N}_{i}\right\} .
$$

It is straightforward to see that the number of fractures, in this case 18 , is equal to $\sum_{i=1}^{m-1}\left|\mathcal{N}_{i}\right|$, where $\left|\mathcal{N}_{i}\right|$ denotes the cardinal of the set $\mathcal{N}_{i}$. In this case, $\mathcal{I}_{1}$ is given by

$$
\begin{aligned}
\mathcal{I}_{1}=\{(1,2),(1,6),(1,7),(1,10),(2,3),(2,7),(2,8),(3,4),(4,5),(4,8),(4,9), \\
\\
(5,6),(5,8),(5,9),(6,7),(6,9),(7,8),(9,10)\} .
\end{aligned}
$$

In order to define the sets of indices $\mathcal{I}_{0}^{T}$ and $\mathcal{I}_{0}^{X}$ that refer to the zero-dimensional intersections of fractures, we will introduce a suitable graph representing the problem. In particular, the graph assigns a node to each subdomain and considers a path connecting the nodes $i$ and $j$ as long as $j \in \mathcal{N}_{i}$ (or, equivalently, whenever the pair of indices $\left.(i, j) \in \mathcal{I}_{1}\right)$. In other words, the graph nodes stand for the subdomains $\left\{\Omega_{i}\right\}_{i=1}^{m}$, and the paths represent the collapsed fractures $\left\{\gamma_{i, j}\right\}_{(i, j) \in \mathcal{I}_{1}}$. Figure 2 (right) shows the graph corresponding to the reduced domain on the left. In this framework, the set of indices $\mathcal{I}_{0}^{T}$ associated to $T$-shaped intersections of three fractures is defined as

$$
\mathcal{I}_{0}^{T}=\left\{(i, j, k) \in \mathbb{N}^{3}: i, j, k \in \mathcal{I}_{2} \text {, with } i<j<k\right. \text {, such that the graph contains }
$$
a closed path passing through the nodes $i, j, k\}$. 
In the example under consideration, the $T$-shaped intersections - marked with a bullet in Figure 2 (left) - are given by the set of indices

$$
\mathcal{I}_{0}^{T}=\{(1,2,7),(1,6,7),(2,7,8),(4,5,8),(4,5,9),(5,6,9)\} .
$$

On the other hand, the set of indices $\mathcal{I}_{0}^{X}$ corresponding to $X$-shaped intersections of four fractures is defined as

$$
\begin{aligned}
\mathcal{I}_{0}^{X}=\{( & (i, j, k, l) \in \mathbb{N}^{4}: i, j, k, l \in \mathcal{I}_{2}, \text { with } i<j<k<l, \text { such that the graph } \\
& \text { contains a closed path passing through the nodes } i, j, k, l, \text { and }(i, j, k), \\
& \left.(i, j, l),(i, k, l),(j, k, l) \notin \mathcal{I}_{0}^{T}\right\} .
\end{aligned}
$$

In the example, the $X$-shaped intersections - marked with a cross in Figure 2 (left) are given by the set of indices

$$
\mathcal{I}_{0}^{X}=\{(2,3,4,8),(5,6,7,8),(1,6,9,10)\} .
$$

Note that the set of indices $(4,5,6,9)$ also defines a closed path in the graph, but $(4,5,9),(5,6,9) \in \mathcal{I}_{0}^{T}$, so it does not represent an $X$-shaped intersection. Something similar applies to the sets of indices $(1,2,6,7),(4,5,8,9)$, and $(1,2,7,8)$.

Finally, let us denote by $\gamma(i)$ the set of all adjacent fractures to subdomain $\Omega_{i}$ for $i \in \mathcal{I}_{2}$. In particular, $\gamma(1)=\left\{\gamma_{1,2}, \gamma_{1,6}, \gamma_{1,7}, \gamma_{1,10}\right\}, \gamma(2)=\left\{\gamma_{1,2}, \gamma_{2,3}, \gamma_{2,7}, \gamma_{2,8}\right\}$ and so on. In turn, $\sigma(i, j)$ is defined as the set of all intersecting points in which the fracture $\gamma_{i, j}$ is involved for $(i, j) \in \mathcal{I}_{1}$. In this case, $\sigma(1,2)=\left\{\sigma_{1,2,7}\right\}, \sigma(1,6)=$ $\left\{\sigma_{1,6,7}, \sigma_{1,6,9,10}\right\}$ and so on.

With the aim of defining an interface problem, each fracture permeability $\mathbf{K}_{i, j}$ is decomposed in a similar way to that introduced in (2.4) with corresponding coefficients $K_{i, j}^{\tau}$ and $K_{i, j}^{\mathbf{n}}$. In this framework, the following mixed-dimensional problem is formulated for $i \in \mathcal{I}_{2}$ and $(i, j) \in \mathcal{I}_{1}[7]$ :

$$
\begin{aligned}
\mathbf{u}_{i} & =-\mathbf{K}_{i} \nabla p_{i} & & \text { in } \Omega_{i}, \\
\nabla \cdot \mathbf{u}_{i} & =q_{i} & & \text { in } \Omega_{i}, \\
\mathbf{u}_{i, j} & =-d_{i, j} K_{i, j}^{\boldsymbol{\tau}} \nabla_{i, j}^{\boldsymbol{\tau}} p_{i, j} & & \text { in } \gamma_{i, j}, \\
\nabla_{i, j}^{\tau} \cdot \mathbf{u}_{i, j} & =q_{i, j}+\left(\mathbf{u}_{i} \cdot \mathbf{n}_{i}+\mathbf{u}_{j} \cdot \mathbf{n}_{j}\right) & & \text { in } \gamma_{i, j}, \\
\alpha_{i, j}\left(p_{i}-p_{i, j}\right) & =\xi \mathbf{u}_{i} \cdot \mathbf{n}_{i}-(1-\xi) \mathbf{u}_{j} \cdot \mathbf{n}_{j} & & \text { in } \gamma_{i, j}, \\
\alpha_{i, j}\left(p_{j}-p_{i, j}\right) & =\xi \mathbf{u}_{j} \cdot \mathbf{n}_{j}-(1-\xi) \mathbf{u}_{i} \cdot \mathbf{n}_{i} & & \text { in } \gamma_{i, j}, \\
p_{i} & =0 & & \text { on } \Gamma_{i}, \\
p_{i, j} & =0 & & \text { on } \Gamma_{i, j},
\end{aligned}
$$

where $\alpha_{i, j}=2 K_{i, j}^{\mathbf{n}} / d_{i, j}$ and $\Gamma_{i, j}=\partial \gamma_{i, j} \cap \Gamma$. The notations $\nabla_{i, j}^{\tau} \cdot$ and $\nabla_{i, j}^{\tau}$ stand for the tangential divergence and gradient operators, as defined by (2.3), on the fracture $\gamma_{i, j}$. At the intersections, we shall impose mass conservation and pressure continuity. In particular, at every $T$-shaped intersecting point $\sigma_{i, j, k}$, with $(i, j, k) \in \mathcal{I}_{0}^{T}$, we impose

$$
\begin{aligned}
\sum_{m, n \in\{i, j, k\},(m, n) \in \mathcal{I}_{1}} \mathbf{u}_{m, n} \cdot \mathbf{n}_{m, n} & =0, \\
p_{m, n} & =p_{i, j, k} \quad \forall m, n \in\{i, j, k\},(m, n) \in \mathcal{I}_{1} .
\end{aligned}
$$


In turn, at every $X$-shaped intersecting point $\sigma_{i, j, k, l}$, with $(i, j, k, l) \in \mathcal{I}_{0}^{X}$, we impose

$$
\begin{aligned}
\sum_{m, n \in\{i, j, k, l\},(m, n) \in \mathcal{I}_{1}} \mathbf{u}_{m, n} \cdot \mathbf{n}_{m, n} & =0, \\
p_{m, n} & =p_{i, j, k, l} \quad \forall m, n \in\{i, j, k, l\},(m, n) \in \mathcal{I}_{1} .
\end{aligned}
$$

For a discussion on more general compatibility conditions at the intersections, we refer the reader to $[29,30,34,69]$.

In order to define the weak formulation of problem (2.10)-(2.12), we introduce the spaces for the velocity unknowns [7]

$$
\begin{aligned}
& \mathbf{W}^{2}=\bigoplus_{i \in \mathcal{I}_{2}}\left\{\mathbf{v}_{i} \in H\left(\operatorname{div}, \Omega_{i}\right): \mathbf{v}_{i} \cdot \mathbf{n}_{i} \in L^{2}(\gamma) \forall \gamma \in \gamma(i)\right\}, \\
& \mathbf{W}^{1}=\bigoplus_{(i, j) \in \mathcal{I}_{1}}\left\{\mathbf{v}_{i, j} \in H\left(\operatorname{div}_{i, j}^{\boldsymbol{\tau}}, \gamma_{i, j}\right): \mathbf{v}_{i, j} \cdot \mathbf{n}_{i, j} \in L^{2}(\sigma) \forall \sigma \in \sigma(i, j)\right\},
\end{aligned}
$$

together with the spaces for the pressures

$$
\begin{aligned}
M^{2} & =\bigoplus_{i \in \mathcal{I}_{2}} L^{2}\left(\Omega_{i}\right), & M^{1} & =\bigoplus_{(i, j) \in \mathcal{I}_{1}} L^{2}\left(\gamma_{i, j}\right), \\
M^{0, T} & =\bigoplus_{(i, j, k) \in \mathcal{I}_{0}^{T}} L^{2}\left(\sigma_{i, j, k}\right), & M^{0, X} & =\bigoplus_{(i, j, k, l) \in \mathcal{I}_{0}^{X}} L^{2}\left(\sigma_{i, j, k, l}\right) .
\end{aligned}
$$

Note that the superscript notation of these spaces provides information about the dimensionality of the corresponding domain in which they are defined. This dimensional decomposition framework has been proposed in [16]. Then the global spaces $\mathbf{W}=\mathbf{W}^{2} \oplus \mathbf{W}^{1}$ and $M=M^{2} \oplus M^{1} \oplus M^{0, T} \oplus M^{0, X}$ are endowed with the norms

$$
\begin{aligned}
\|\mathbf{v}\|_{\mathbf{W}}^{2}= & \sum_{i \in \mathcal{I}_{2}}\left(\left\|\mathbf{v}_{i}\right\|_{L^{2}\left(\Omega_{i}\right)}^{2}+\left\|\nabla \cdot \mathbf{v}_{i}\right\|_{L^{2}\left(\Omega_{i}\right)}^{2}+\sum_{\gamma \in \gamma(i)}\left\|\mathbf{v}_{i} \cdot \mathbf{n}_{i}\right\|_{L^{2}(\gamma)}^{2}\right) \\
& +\sum_{(i, j) \in \mathcal{I}_{1}}\left(\left\|\mathbf{v}_{i, j}\right\|_{L^{2}\left(\gamma_{i, j}\right)}^{2}+\left\|\nabla_{i, j}^{\tau} \cdot \mathbf{v}_{i, j}\right\|_{L^{2}\left(\gamma_{i, j}\right)}^{2}+\sum_{\sigma \in \sigma(i, j)}\left\|\mathbf{v}_{i, j} \cdot \mathbf{n}_{i, j}\right\|_{L^{2}(\sigma)}^{2}\right), \\
\|r\|_{M}^{2}= & \sum_{i \in \mathcal{I}_{2}}\left\|r_{i}\right\|_{L^{2}\left(\Omega_{i}\right)}^{2}+\sum_{(i, j) \in \mathcal{I}_{1}}\left\|r_{i, j}\right\|_{L^{2}\left(\gamma_{i, j}\right)}^{2}+\sum_{(i, j, k) \in \mathcal{I}_{0}^{T}}\left\|r_{i, j, k}\right\|_{L^{2}\left(\sigma_{i, j, k}\right)}^{2} \\
& +\sum_{(i, j, k, l) \in \mathcal{I}_{0}^{X}}\left\|r_{i, j, k, l}\right\|_{L^{2}\left(\sigma_{i, j, k, l}\right)}^{2} .
\end{aligned}
$$

In this framework, the bilinear forms $a: \mathbf{W} \times \mathbf{W} \rightarrow \mathbb{R}$ and $b: \mathbf{W} \times M \rightarrow \mathbb{R}$ are given, respectively, by

$$
\begin{aligned}
a(\mathbf{u}, \mathbf{v})= & \sum_{i \in \mathcal{I}_{2}}\left(\mathbf{K}_{i}^{-1} \mathbf{u}_{i}, \mathbf{v}_{i}\right)_{\Omega_{i}}+\sum_{(i, j) \in \mathcal{I}_{1}}\left(\left(d_{i, j} K_{i, j}^{\boldsymbol{\tau}}\right)^{-1} \mathbf{u}_{i, j}, \mathbf{v}_{i, j}\right)_{\gamma_{i, j}} \\
& +\sum_{(i, j) \in \mathcal{I}_{1}}\left(\alpha_{i, j}^{-1}\left(\xi \mathbf{u}_{i} \cdot \mathbf{n}_{i}-(1-\xi) \mathbf{u}_{j} \cdot \mathbf{n}_{j}\right), \mathbf{v}_{i} \cdot \mathbf{n}_{i}\right)_{\gamma_{i, j}} \\
& +\sum_{(i, j) \in \mathcal{I}_{1}}\left(\alpha_{i, j}^{-1}\left(\xi \mathbf{u}_{j} \cdot \mathbf{n}_{j}-(1-\xi) \mathbf{u}_{i} \cdot \mathbf{n}_{i}\right), \mathbf{v}_{j} \cdot \mathbf{n}_{j}\right)_{\gamma_{i, j}}
\end{aligned}
$$


and

$$
\begin{aligned}
b(\mathbf{u}, r)= & \sum_{i \in \mathcal{I}_{2}}\left(\nabla \cdot \mathbf{u}_{i}, r_{i}\right)_{\Omega_{i}}+\sum_{(i, j) \in \mathcal{I}_{1}}\left(\nabla_{i, j}^{\boldsymbol{\tau}} \cdot \mathbf{u}_{i, j}, r_{i, j}\right)_{\gamma_{i, j}} \\
& -\sum_{(i, j) \in \mathcal{I}_{1}}\left(\mathbf{u}_{i} \cdot \mathbf{n}_{i}+\mathbf{u}_{j} \cdot \mathbf{n}_{j}, r_{i, j}\right)_{\gamma_{i, j}} \\
& -\sum_{(i, j, k) \in \mathcal{I}_{0}^{T}}\left(\sum_{(m, n) \in\{i, j, k\},(m, n) \in \mathcal{I}_{1}} \mathbf{u}_{m, n} \cdot \mathbf{n}_{m, n}, r_{i, j, k}\right)_{\sigma_{i, j, k}} \\
& -\sum_{(i, j, k, l) \in \mathcal{I}_{0}^{X}}\left(\sum_{(m, n) \in\{i, j, k, l\},(m, n) \in \mathcal{I}_{1}} \mathbf{u}_{m, n} \cdot \mathbf{n}_{m, n}, r_{i, j, k, l}\right)_{\sigma_{i, j, k, l}} .
\end{aligned}
$$

In turn, the linear form $L: M \rightarrow \mathbb{R}$ associated with the source terms is defined as

$$
L(r)=\sum_{i \in \mathcal{I}_{2}}\left(q_{i}, r_{i}\right)_{\Omega_{i}}+\sum_{(i, j) \in \mathcal{I}_{1}}\left(q_{i, j}, r_{i, j}\right)_{\gamma_{i, j}} .
$$

In this setting, the weak formulation of problem (2.10)-(2.12) shows the same structure as (2.6). However, since the function spaces and forms are newly defined in this case, we reproduce it here for convenience: Find $(\mathbf{u}, p) \in \mathbf{W} \times M$ such that

$$
\begin{aligned}
a(\mathbf{u}, \mathbf{v})-b(\mathbf{v}, p) & =0 & & \forall \mathbf{v} \in \mathbf{W}, \\
b(\mathbf{u}, r) & =L(r) & & \forall r \in M .
\end{aligned}
$$

Following [7, 52], if the permeabilities in the subdomains and the coefficients $K_{i, j}^{\mathbf{n}} / d_{i, j}$ and $K_{i, j}^{\tau} d_{i, j}$ are bounded by positive constants, the existence and uniqueness of solution of this problem can be proved for the case $\xi>1 / 2$.

In the rest of this subsection, we introduce a mixed finite element discretization for problem (2.13). Along the lines of subsection 2.3, we suppose that the subdomains $\Omega_{i}$ admit a rectangular partition $\mathcal{T}_{i}^{h}$ for $i \in \mathcal{I}_{2}$. Further, the meshes $\mathcal{T}_{i}^{h}$ are assumed to match at the interfaces $\gamma_{i, j}$; i.e., they induce a unique partition $\mathcal{T}_{i, j}^{h}$ on $\gamma_{i, j}$ for $(i, j) \in \mathcal{I}_{1}$. Note that the intersecting points of the fractures $\left\{\sigma_{i, j, k}\right\}_{(i, j, k) \in \mathcal{I}_{0}^{T}}$ and $\left\{\sigma_{i, j, k, l}\right\}_{(i, j, k, l) \in \mathcal{I}_{0}^{X}}$ are vertices of some of the preceding meshes. For instance, given $(i, j, k) \in \mathcal{I}_{0}^{T}$, the intersecting point $\sigma_{i, j, k}$ is a vertex of the two-dimensional meshes $\mathcal{T}_{i}^{h}, \mathcal{T}_{j}^{h}$, and $\mathcal{T}_{k}^{h}$ and also a vertex of the one-dimensional meshes $\mathcal{T}_{i, j}^{h}, \mathcal{T}_{j, k}^{h}$, and $\mathcal{T}_{i, k}^{h}$.

In this context, let $\mathbf{W}_{i}^{h} \times M_{i}^{h}$ be the lowest order Raviart-Thomas mixed finite element spaces defined on $\mathcal{T}_{i}^{h}$ for $i \in \mathcal{I}_{2}$; let $\mathbf{W}_{i, j}^{h} \times M_{i, j}^{h}$ be the lowest-order RaviartThomas mixed finite element spaces defined on $\mathcal{T}_{i, j}^{h}$ for $(i, j) \in \mathcal{I}_{1}$; and let $M_{i, j, k}^{h}$ and $M_{i, j, k, l}^{h}$ be equal to $\mathbb{R}$ for $(i, j, k) \in \mathcal{I}_{0}^{T}$ and $(i, j, k, l) \in \mathcal{I}_{0}^{X}$. Based on the notations

$$
\begin{aligned}
\mathbf{W}^{h, 2} & =\bigoplus_{i \in \mathcal{I}_{2}} \mathbf{W}_{i}^{h}, & \mathbf{W}^{h, 1} & =\bigoplus_{(i, j) \in \mathcal{I}_{1}} \mathbf{W}_{i, j}^{h}, \\
M^{h, 2} & =\bigoplus_{i \in \mathcal{I}_{2}} M_{i}^{h}, & M^{h, 1} & =\bigoplus_{(i, j) \in \mathcal{I}_{1}} M_{i, j}^{h}, \\
M^{h, 0, T} & =\bigoplus_{(i, j, k) \in \mathcal{I}_{0}^{T}} M_{i, j, k}^{h}, & M^{h, 0, X} & =\bigoplus_{(i, j, k, l) \in \mathcal{I}_{0}^{X}} M_{i, j, k, l}^{h},
\end{aligned}
$$


the approximation spaces are obtained as $\mathbf{W}^{h}=\mathbf{W}^{h, 2} \oplus \mathbf{W}^{h, 1}$ for the velocities and $M^{h}=M^{h, 2} \oplus M^{h, 1} \oplus M^{h, 0, T} \oplus M^{h, 0, X}$ for the pressures. Then, using the quadrature rules introduced in the previous subsection, we define the discrete forms

$$
\begin{aligned}
a_{h}(\mathbf{u}, \mathbf{v})= & \sum_{i \in \mathcal{I}_{2}}\left(\mathbf{K}_{i}^{-1} \mathbf{u}_{i}, \mathbf{v}_{i}\right)_{\Omega_{i}, \mathbf{T M}}+\sum_{(i, j) \in \mathcal{I}_{1}}\left(\left(d_{i, j} K_{i, j}^{\boldsymbol{\tau}}\right)^{-1} \mathbf{u}_{i, j}, \mathbf{v}_{i, j}\right)_{\gamma_{i, j}, \mathbf{T}} \\
& +\sum_{(i, j) \in \mathcal{I}_{1}}\left(\alpha_{i, j}^{-1}\left(\xi \mathbf{u}_{i} \cdot \mathbf{n}_{i}-(1-\xi) \mathbf{u}_{j} \cdot \mathbf{n}_{j}\right), \mathbf{v}_{i} \cdot \mathbf{n}_{i}\right)_{\gamma_{i, j}} \\
& +\sum_{(i, j) \in \mathcal{I}_{1}}\left(\alpha_{i, j}^{-1}\left(\xi \mathbf{u}_{j} \cdot \mathbf{n}_{j}-(1-\xi) \mathbf{u}_{i} \cdot \mathbf{n}_{i}\right), \mathbf{v}_{j} \cdot \mathbf{n}_{j}\right)_{\gamma_{i, j}}, \\
L_{h}(r)= & \sum_{i \in \mathcal{I}_{2}}\left(q_{i}, r_{i}\right)_{\Omega_{i}, \mathbf{M}}+\sum_{(i, j) \in \mathcal{I}_{1}}\left(q_{i, j}, r_{i, j}\right)_{\gamma_{i, j}, \mathbf{M}} .
\end{aligned}
$$

Finally, the mixed finite element approximation to (2.13) takes the form (2.7) for the newly defined discrete spaces and forms: Find $\left(\mathbf{u}_{h}, p_{h}\right) \in \mathbf{W}^{h} \times M^{h}$ such that

$$
\begin{aligned}
a_{h}\left(\mathbf{u}_{h}, \mathbf{v}_{h}\right)-b\left(\mathbf{v}_{h}, p_{h}\right) & =0 & & \forall \mathbf{v}_{h} \in \mathbf{W}^{h}, \\
b\left(\mathbf{u}_{h}, r_{h}\right) & =L_{h}\left(r_{h}\right) & & \forall r_{h} \in M^{h} .
\end{aligned}
$$

In order to derive the algebraic linear system underlying (2.14), we group the pressure and velocity unknowns taking into account their dimensions, i.e., $\mathbf{u}_{h}=\left[U^{2}, U^{1}\right]^{T}$ and $p_{h}=\left[P^{2}, P^{1}, P^{0}\right]^{T}$. Note that $U^{2}$ and $U^{1}$ are vectors related to the velocity unknowns on the two-dimensional subdomains and the one-dimensional fractures, respectively. In turn, $P^{2}, P^{1}$, and $P^{0}$ are vectors related to the pressure unknowns on the two-dimensional subdomains, the one-dimensional fractures, and the zerodimensional intersections, respectively. In this context, the algebraic linear system stemming from (2.14) may be written as the saddle-point problem

$$
\left[\begin{array}{ccccc}
A_{2,2} & 0 & B_{2,2}^{T} & F_{2,1}^{T} & 0 \\
0 & A_{1,1} & 0 & B_{1,1}^{T} & F_{1,0}^{T} \\
B_{2,2} & 0 & 0 & 0 & 0 \\
F_{2,1} & B_{1,1} & 0 & 0 & 0 \\
0 & F_{1,0} & 0 & 0 & 0
\end{array}\right]\left[\begin{array}{c}
U^{2} \\
U^{1} \\
P^{2} \\
P^{1} \\
P^{0}
\end{array}\right]=\left[\begin{array}{c}
0 \\
0 \\
Q^{2} \\
Q^{1} \\
0
\end{array}\right]
$$

This is a generalization of the linear system (2.9) obtained in the case of a single fracture. In such a case, $U^{2}$ and $P^{2}$ were composed of two blocks, one per subdomain, while $U^{1}$ and $P^{1}$ consisted of one block corresponding to the only fracture $\gamma$ and $P^{0}$ was lacking since there were no intersecting points. In contrast, for the example shown in Figure 2, $U^{2}$ and $P^{2}$ would be composed of 10 blocks, one per subdomain; $U^{1}$ and $P^{1}$ would consist of 18 blocks, one per fracture; and $P^{0}$ would group 9 pressure unknowns, one per intersecting point of fractures.

3. Monolithic multigrid methods for mixed-dimensional elliptic problems. In this work, we propose an efficient monolithic geometric multigrid solver for flow in fractured porous media. Multigrid methods [17, 78] aim to accelerate the slow convergence of classical iterative methods by using coarse meshes. Since these latter have a strong smoothing effect on the error of the solution, this error can be properly represented in coarser grids where the computations are much less expensive. 


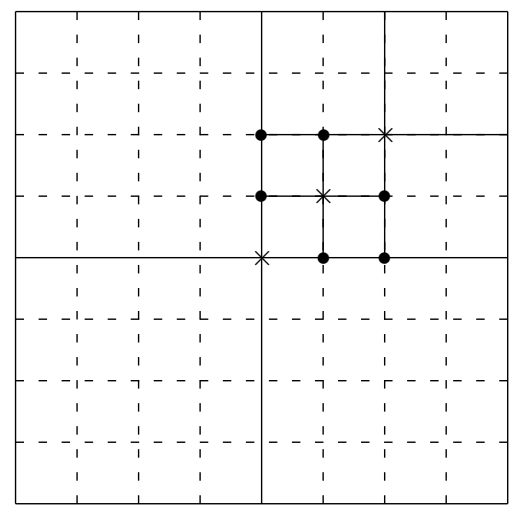

FIG. 3. Coarsest grid corresponding to the fracture network shown in Figure 2.

Suppose that $A_{k} u_{k}=f_{k}$ is the system to solve, where the matrix $A_{k}$ corresponds to a discretization of a partial differential equation on a grid $G^{k}, f_{k}$ is the right-hand side, and $u_{k}$ is the unknown vector. In order to apply a standard two-grid cycle for solving this problem, we perform the following steps:

1. Apply $\nu_{1}$ iterations of a classical iterative method, called smoother, on $G^{k}$ (pre-smoothing step).

2. Compute the residual of the current fine grid approximation.

3. Restrict the residual to the coarse grid $G^{k-1}$ by using a restriction operator $R_{k}^{k-1}$.

4. Solve the residual equation on the coarse grid.

5. Interpolate the obtained correction to the fine grid $G^{k}$ by using a prolongation operator $P_{k-1}^{k}$.

6. Add the interpolated correction to the current fine grid approximation.

7. Apply $\nu_{2}$ iterations of a classical iterative method on $G^{k}$ (post-smoothing step).

Since we do not need to solve the problem on the coarse grid exactly, we can apply the same algorithm in a recursive way by using a hierarchy of coarser meshes, giving rise to the well-known multigrid cycle. It is clear that many details have to be fixed for the design of an efficient multigrid method since all the components have to be properly chosen. In particular, we need to specify the hierarchy of grids, the coarsegrid operators, the type of cycle, the intergrid transfer operators, and the smoothing procedure. Next, we explain our choices in this work.

3.1. Hierarchy of meshes, coarse-grid operators, and cycle type. The implementation of a geometric multigrid method requires defining the problem on grids of various sizes, namely, a hierarchy of grids. Here, such a hierarchy is constructed in the following way. First, we consider a coarse grid which is built taking into account the location of the fractures. This mesh is generated by assuming that every fracture coincides with an edge of some element in the porous medium grid. As explained in section 2, we suppose that the grids in the subdomains match at the interfaces. Thus, in the case of considering $m$ subdomains, we define $G^{0}=\bigcup_{i=1}^{m} \mathcal{T}_{i}^{h}$ as the coarsest possible grid fulfilling this criterion. As an example, if we consider the fracture configuration shown in Figure 2, the coarsest grid $G^{0}$ is given in Figure 3.

Then the hierarchy of computational grids is created by applying a regular refinement process to each cell in that initial mesh. This means that we obtain a sequence 


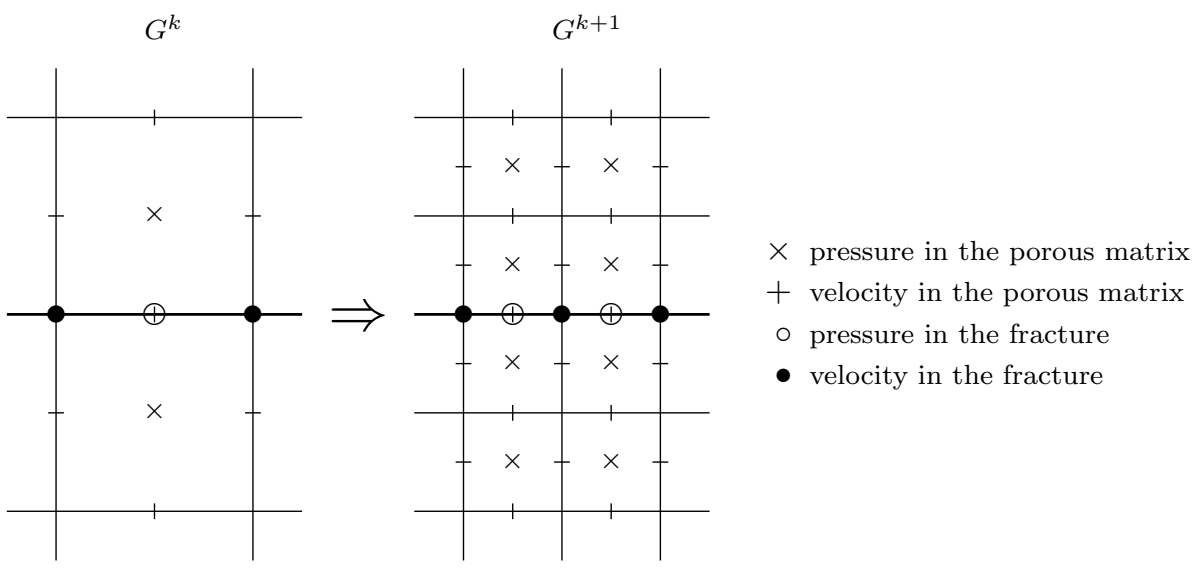

FIG. 4. Grid refinement procedure and location of the unknowns for both porous matrix and fracture (in bold line).

of successively finer grids $G^{0}, G^{1}, \ldots, G^{M}$. In particular, since we are considering a quadrilateral partition of the porous medium, $G^{k+1}$ is obtained from $G^{k}$ by dividing each cell into four new elements for the next finer grid, as shown in Figure 4, and this process continues until a fine enough target grid $G^{M}$ is obtained.

Once the mesh hierarchy is generated, we consider a direct discretization of our problem on each grid. As for the type of cycle, we use W-cycles since we have seen that this choice gives very good results for solving difficult coupled problems like the Darcy-Stokes system [48] and the Biot-Stokes system [49].

3.2. Intergrid transfer operators. Now we define the restriction and interpolation operators involved in the multigrid method for solving the mixed-dimensional problem. We consider different transfer operators for the unknowns belonging to the matrix and for those located at the fractures. In particular, we choose two-dimensional and one-dimensional transfer operators, respectively. This means that we implement mixed-dimensional transfer operators in our multigrid algorithm in order to handle the problem at once. In matrix form, the chosen restriction operator $R_{k}^{k-1}$ from grid $G^{k}$ to $G^{k-1}$ is a block diagonal matrix since the velocity and pressure variables are uncoupled, and the unknowns in the porous matrix and those in the fractures are uncoupled too.

Due to the use of quadrature rules in this work, the mixed finite element method turns into a finite difference scheme on a staggered grid. As a consequence, we consider the standard restriction operators used for this type of mesh. Regarding the unknowns of the porous medium, we take into account the staggered arrangement of their location. Thus, the intergrid transfer operators that act in the porous media unknowns are defined as follows: a six-point restriction is considered at velocity grid points, and a four-point restriction is applied at pressure grid points, as can be seen in Figure 5. The prolongation operator $P_{k-1}^{k}$ is chosen to be the adjoint of the restriction.

Regarding the intergrid transfer operators for the unknowns at the fractures, we again take into account their one-dimensional staggered arrangement, yielding the restriction transfer operators shown in Figure 6. Finally, the prolongation operators are chosen to be the corresponding adjoints. 

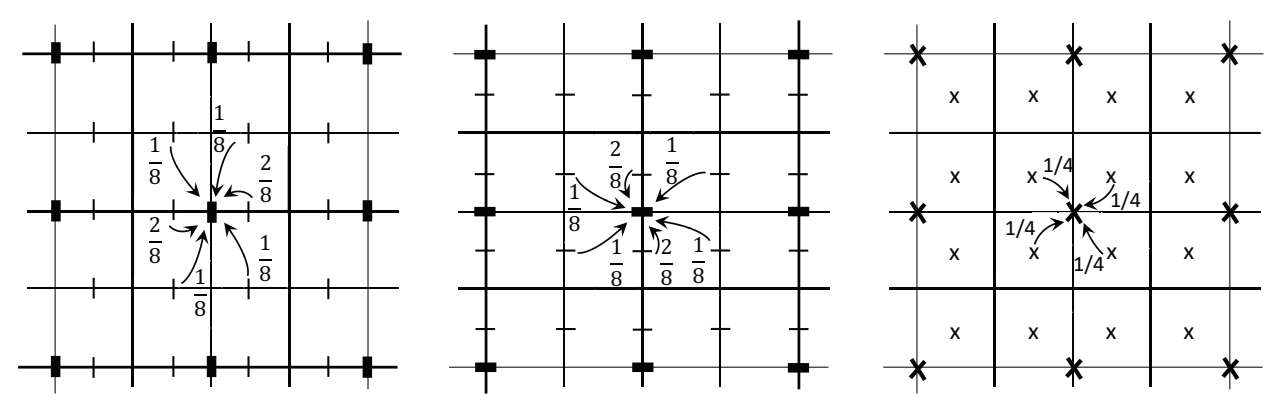

FIG. 5. Restriction operators acting at the porous media unknowns.
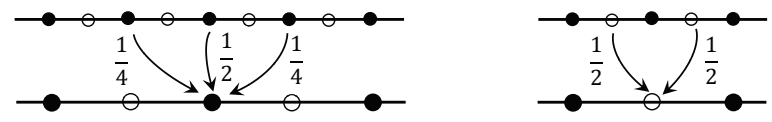

FIG. 6. Restriction operators acting at the fracture unknowns.

3.3. Smoother. The performance of a multigrid method is essentially influenced by the smoothing algorithm. Here, in order to deal with the difficulties generated by a saddle-point problem, we consider a relaxation iteration among the class of multiplicative Schwarz smoothers. Basically, this type of iteration can be described as an overlapping block Gauss-Seidel method, where a small linear system of equations for each grid point has to be solved at each smoothing step. A particular case of such relaxation schemes is the so-called Vanka smoother, introduced in [79] for solving the staggered finite difference discretization of the Navier-Stokes equations.

Due to the mixed-dimensional character of our problem, we propose a smoother $S_{h}=S_{h}^{0} S_{h}^{1} S_{h}^{2}$ which is written as the composition of three relaxation procedures acting on the two-dimensional cells of the porous matrix $\left(S_{h}^{2}\right)$, the one-dimensional elements in the fractures $\left(S_{h}^{1}\right)$, and the zero-dimensional intersection points $\left(S_{h}^{0}\right)$. Next, we describe these partial relaxation procedures.

1. Relaxation for the porous matrix. The smoother considered for the unknowns located outside the fractured part of the domain is based on simultaneously updating all the unknowns appearing in the discrete divergence operator in the pressure equation. This way of building the blocks is very common in the Vanka-type smoothers used for Stokes and Navier-Stokes problems. This approach implies that four unknowns corresponding to velocities and one pressure unknown (see Figure 7(a)) are relaxed simultaneously, making it necessary to solve a $5 \times 5$ system for each cell. Then we iterate over all the elements in lexicographic order, and for each of them the corresponding box is solved.

2. Relaxation for the fractures. The relaxation step applied to the unknowns located at the fractures is again based on simultaneously updating all the unknowns appearing in the discrete divergence operator in the pressure equation. This means that, in this case, for each element in the fracture we update five unknowns: three of them corresponding to the fracture and two of them to the matrix. In particular, each pressure unknown in the fracture is updated together with the two fracture velocities within the same element and the two porous matrix velocities located at the edges of the corresponding 


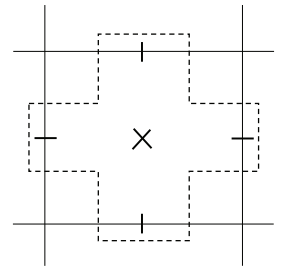

(a)

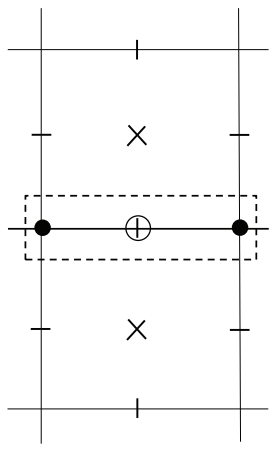

(b) $\times$ pressure in the porous matrix

+ velocity in the porous matrix

$\circ$ pressure in the fracture

- velocity in the fracture

FIG. 7. Unknowns updated together by the vanka-type smoothers applied (a) outside the fractures and (b) within the fractures.

two-dimensional elements that match with that particular fracture element. This can be seen in Figure 7(b). Notice that there are three unknowns located at the same point: the pressure in the fracture and the two velocities corresponding to the elements adjacent to the fracture.

3. Relaxation for the intersections. At the intersection points of the fractures we apply a block Gauss-Seidel smoother coupling the fracture velocity unknowns located at each intersection so that we need to solve a $2 \times 2,3 \times 3$, or $4 \times 4$ system of equations on each of these grid points.

The previously defined partial relaxation procedures can be formally written as

$$
S_{h}^{n}=\prod_{B=1}^{N_{B, n}}\left(I-V_{B, n}^{T}\left(A^{B, n}\right)^{-1} V_{B, n} A\right) \quad \text { for } n=0,1,2
$$

where $A$ is the system matrix in (2.15), $N_{B, n}$ is the number of $n$-dimensional elements in the partition, $V_{B, n}$ represents the projection operator from the unknown vector to the vector of unknowns involved in the block to solve, and matrix $A^{B, n}$ is defined as $A^{B, n}=V_{B, n} A V_{B, n}^{T}$.

3.4. Implementation and computational cost. The proposed monolithic mixed-dimensional multigrid method is implemented in a blockwise manner. Given an arbitrary fracture network composed of vertical and horizontal fractures, the first step is to construct a uniform rectangular coarse grid so that the fracture network is contained in the set of edges of the grid. After that, a regular refinement process is applied on each block in the coarse grid until a target mesh with an appropriate fine grid scale to solve the problem is obtained. Then, for each step of the multigrid method, the two-dimensional components are performed in the porous matrix grid points, whereas within the fractures one-dimensional components are implemented (notice that in the smoother, for example, this one-dimensional computation includes also unknowns from the porous matrix). In particular, in the smoother, first the unknowns in the porous matrix are relaxed by using the standard two-dimensional Vanka smoother for the Darcy problem, and, after that, a one-dimensional Vanka smoother is used to update the unknowns located within the fractures. Finally, at the intersection points between different fractures, the velocities from different fractures are simultaneously relaxed. 
The computational cost of an algorithm is an important indicator of the efficiency of the method. Within the framework of multigrid methods, it is well known that, in general, the most time-consuming part is the relaxation procedure. In particular, for the mixed-dimensional multigrid method proposed here, the most costly component is the Vanka-type smoother in the two-dimensional porous matrix due to the fact that it has to solve small dense $5 \times 5$ systems of equations for each pressure grid point. Therefore, the complexity of the proposed two-dimensional Vanka smoother will give us a good approximation of the computational cost of the multigrid method. Within this smoothing iteration, the most expensive parts are the matrix-vector multiplications required to calculate the local residual and the solution of each dense system. The cost of the calculation of each local defect is $\mathcal{O}(n)$, where $n$ is the total number of degrees of freedom. The cost of solving the system depends on the chosen method: if the inverse of the system matrix or the resulting $L$ and $U$ factors in a block $L U$ factorization are stored, the cost of calculating the correction is $\mathcal{O}(n)$. Consequently, the computational cost per iteration of the Vanka-type relaxation is $\mathcal{O}(n)$.

Remark 2. Notice that this strategy can be easily extended to triangular grids in order to deal with more complex fracture networks. The idea would be to construct an unstructured coarse triangulation in such a way that the fracture network is part of its edges and then to apply a regular refinement to the input triangles in order to obtain a semi-structured triangular grid in which the geometric multigrid method can be easily applied (see $[62,63]$ ).

4. Numerical results. In this section, we will demonstrate the robustness of the proposed monolithic mixed-dimensional multigrid method through different numerical experiments. In addition to seeing that the behavior of multigrid is independent of the spatial discretization parameter, we will also analyze the robustness of the algorithm with respect to fracture properties as the permeability. We will consider the proposed multigrid method as a stand-alone solver and as a preconditioner for a Krylov subspace iteration. Further, we will study how the multigrid performance is influenced by considering several fractures and also illustrate the good behavior of the method on a benchmark problem from the literature. Throughout the whole section, we will consider $\xi=1$ in the model, and we will use a $W$-cycle with two pre- and two post-smoothing steps since this choice has been shown to provide very good results when monolithic multigrid solvers are considered for coupled problems [48, 49]. In our case, we will see that it gives multigrid textbook efficiency [78]. All the numerical computations have been carried out on a MacBook Pro with a Core i5 2.7 GHz processor and 8 GB RAM running OS X 10.10511 (Yosemite).

4.1. One-fracture test. We first consider a test problem presented in [52] in which the domain consists of an horizontal rectangular slice of porous medium $\Omega=$ $(0,2) \times(0,1)$ with impermeable bottom and top boundaries and a prescribed pressure of zero and one in the left and right sides, respectively. For the permeability of the porous medium, we consider that $\mathbf{K}=K \mathbf{I}$ with $\mathbf{I}$ the identity tensor. We will study the case of unit permeability $(K=1)$, and we will also perform some tests considering a heterogeneous random medium. Such domain is divided into two equally sized subdomains by a vertical fracture of width $d=10^{-2}$ for which we consider two different cases: constant permeability in the whole fracture and different values of the permeability within the fracture. Also, two different types of boundary conditions are considered in the extremities of the fracture. All these settings are displayed in Figure 8 for both Case 1 and Case 2 . 


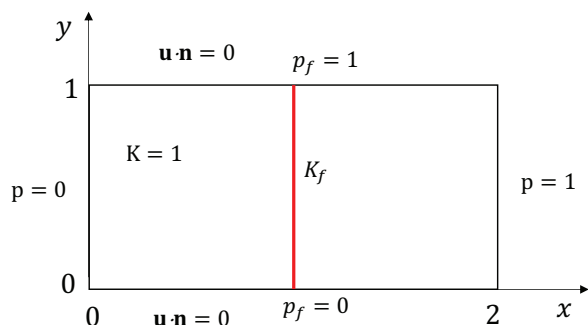

(a) Case 1

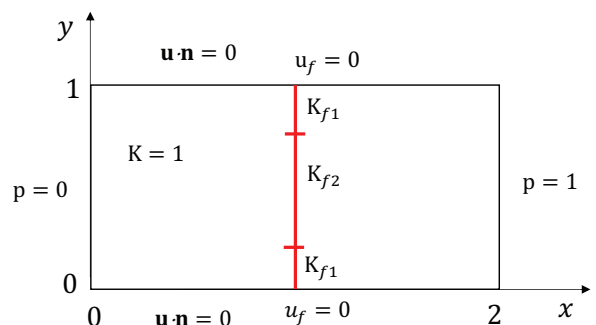

(b) Case 2

FIG. 8. Domain and boundary conditions for the first numerical experiment.

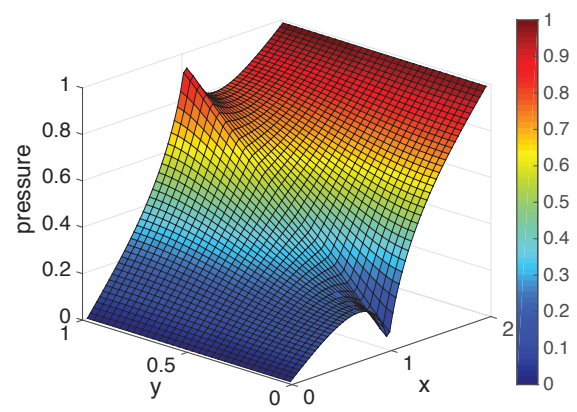

(a)

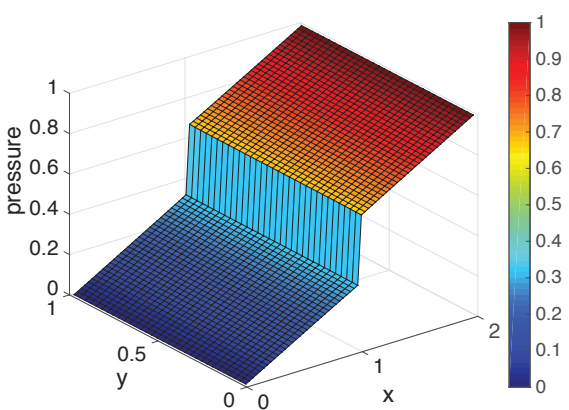

(b)

FIG. 9. Pressure solution for the fracture permeabilities (a) $K_{f}=100$ and (b) $K_{f}=0.01$ for the first numerical experiment (Case 1).

4.1.1. Case 1: Constant permeability in the fracture. In this first case we consider the setting displayed in Figure 8(a). The boundary conditions for the fracture are Dirichlet in this case. More concretely, $p_{f}=1$ on the top extremity of the fracture, and $p_{f}=0$ on the bottom. The permeability tensor in the fracture is given by $\mathbf{K}_{f}=K_{f} \mathbf{I}$, and we want to study the influence of different values of $K_{f}$ on the performance of the multigrid solver. We consider both conductive fractures and blocking fractures, characterized by high or low permeabilities, respectively. As an example, in Figure 9 we show the pressure solution obtained for two different values of $K_{f}$ : one representative of a high permeability (left side) and the other one characteristic of a low permeability (right side) in the fracture.

Now, to study the robustness of the mixed-dimensional multigrid method with respect to different values of the permeability, in Table 1 we display the number of iterations needed to reduce the initial residual in a factor of $10^{-10}$ for different grid sizes and for low and high permeabilities.

We can observe that, for all the values of $K_{f}$, the performance of the multigrid method is independent of the spatial discretization parameter. Moreover, only a few iterations are required to satisfy the stopping criterion. In Table 1 we also present the number of iterations needed to solve this first test case by using the proposed mixeddimensional multigrid method as preconditioner of the flexible GMRES (FGMRES) method. In this case, applying the multigrid method as preconditioner means a single $W(2,2)$-multigrid iteration. The numbers of iterations needed by the preconditioned 
TABLE 1

Number of iterations necessary to solve the first numerical experiment (Case 1) with different values of a constant permeability tensor in the fracture by using the proposed multigrid method as stand-alone solver ( $M G)$ and as preconditioner (Prec).

\begin{tabular}{ccccccc} 
& & $32 \times 16$ & $64 \times 32$ & $128 \times 64$ & $256 \times 128$ & $512 \times 256$ \\
\cline { 2 - 6 } & $K_{f}$ & MG/Prec & MG/Prec & MG/Prec & MG/Prec & MG/Prec \\
\hline \multirow{2}{*}{ Low } & $10^{-6}$ & $8 / 8$ & $8 / 8$ & $9 / 9$ & $9 / 9$ & $9 / 9$ \\
permeability & $10^{-4}$ & $8 / 8$ & $8 / 8$ & $9 / 9$ & $9 / 9$ & $9 / 9$ \\
& $10^{-2}$ & $8 / 8$ & $8 / 8$ & $9 / 9$ & $9 / 9$ & $9 / 9$ \\
\hline \hline \multirow{2}{*}{ High } & $10^{2}$ & $10 / 9$ & $9 / 9$ & $9 / 9$ & $10 / 9$ & $10 / 10$ \\
permeability & $10^{4}$ & $8 / 9$ & $9 / 9$ & $9 / 10$ & $9 / 10$ & $10 / 10$ \\
& $10^{6}$ & $8 / 9$ & $9 / 9$ & $9 / 10$ & $9 / 10$ & $10 / 10$ \\
\hline
\end{tabular}
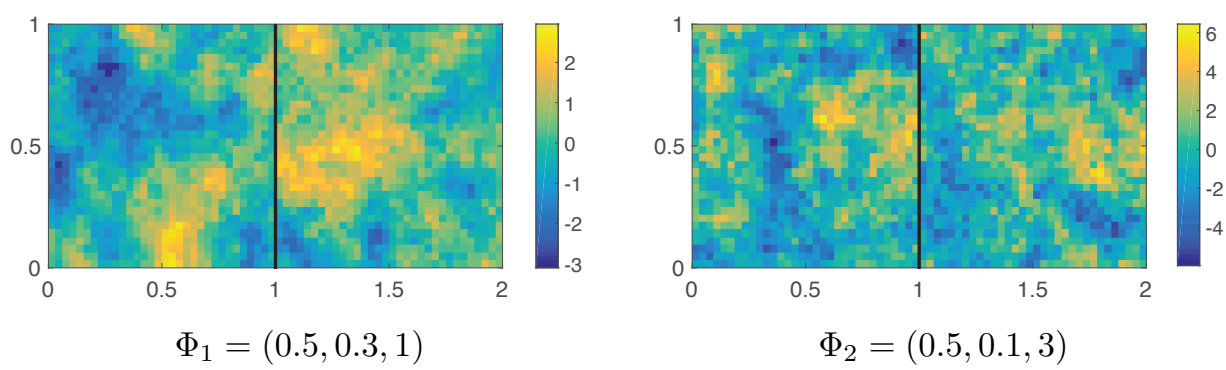

FIG. 10. Logarithm of the permeability in the porous matrix, generated using two different sets of parameters $\Phi=\left(\nu_{c}, \lambda_{c}, \sigma_{c}^{2}\right)$.

FGMRES are almost identical to those obtained with the direct application of the multigrid solver.

The application of the proposed multigrid as preconditioner seems more appealing when dealing with a heterogeneous porous medium. Thus, next we consider the same example in which the permeability in the porous matrix is represented by a lognormal random field; that is, the logarithm of the permeability, $\log _{10} K$, is modeled by a zeromean Gaussian random field. In order to generate samples of the Gaussian random field, we consider the Matérn covariance function

$$
C_{\Phi}(r)=\sigma_{c}^{2} \frac{2^{1-\nu_{c}}}{\Gamma\left(\nu_{c}\right)}\left(2 \sqrt{\nu_{c}} \frac{r}{\lambda_{c}}\right)^{\nu_{c}} K_{\nu}\left(2 \sqrt{\nu_{c}} \frac{r}{\lambda_{c}}\right)
$$

which is characterized by the set of parameters $\Phi=\left(\nu_{c}, \lambda_{c}, \sigma_{c}^{2}\right)$ that denote the field smoothness, the correlation length, and the variance, respectively. Argument $r$ of function $C_{\Phi}$ represents the distance between two points, $\Gamma$ is the gamma function, and $K_{\nu}$ is the modified Bessel function of the second kind. For our study, we consider two Matérn reference parameter sets $\Phi_{1}=(0.5,0.3,1)$ and $\Phi_{2}=(0.5,0.1,3)$ with increasing complexity, and in Figure 10 we display an example of the logarithm of the permeability for each of the parameter sets. It is clearly observed that for the more complex set, $\Phi_{2}$, the fluctuations of the permeability field are much larger than for $\Phi_{1}$.

When applying the FGMRES method preconditioned with the proposed mixeddimensional multigrid algorithm until the initial residual is reduced in a factor of $10^{-10}$, we obtain an average number of 9 iterations for the parameter set $\Phi_{1}$ and around 13 iterations when the more difficult set $\Phi_{2}$ is considered. In both cases, we 


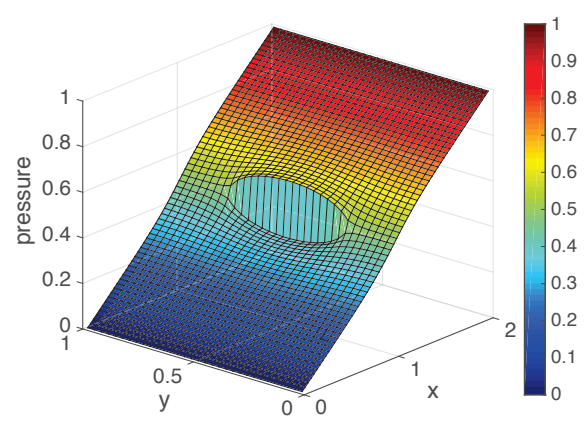

(a)

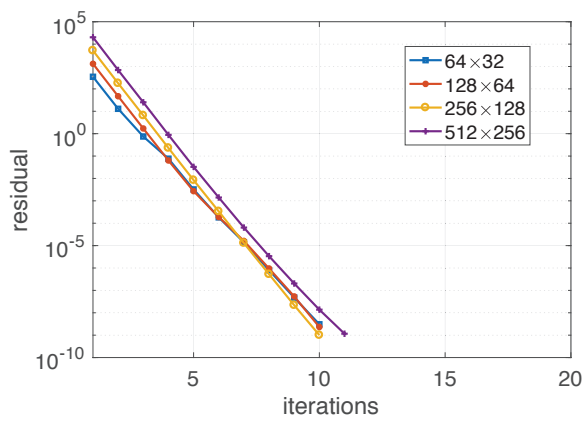

(b)

FIG. 11. (a) Pressure solution and (b) history of the convergence of the proposed multigrid method for the first numerical experiment (Case 2).

obtain a preconditioner with a robust convergence independently of the discretization parameter $h$.

4.1.2. Case 2: Variable permeability in the fracture. We consider now the setting displayed in Figure 8(b). In this case, the boundary conditions for the fracture are homogeneous Neumann conditions on both extremities of the fracture. The permeability tensor in the fracture is now given by

$$
\mathbf{K}_{f}= \begin{cases}K_{f 1} \mathbf{I} & \text { if } 0<y<\frac{1}{4} \text { or } \frac{3}{4}<y<1, \\ K_{f 2} \mathbf{I} & \text { if } \frac{1}{4}<y<\frac{3}{4} .\end{cases}
$$

In particular, we consider $K_{f 1}=10^{2}$ and $K_{f 2}=2 \times 10^{-3}$. Due to the low value of $K_{f 2}$ the fluid tends to avoid the middle part of the fracture, representing a geological barrier. This behavior can be clearly seen in the pressure distribution depicted in Figure 11(a).

Finally, we want to study if these changes of permeability within the fracture have some effect on the multigrid performance. For this purpose, in Figure 11(b) we display the history of the convergence of the multigrid solver for different mesh sizes. More concretely, the reduction of the residual is depicted against the number of iterations, and the stopping criterion is to reduce the initial residual until $10^{-8}$. It is clearly seen that the convergence of the monolithic mixed-dimensional multigrid method is independent of the spatial discretization parameter, and the number of iterations are very similar to those obtained in the previous case with a constant permeability in the fracture.

4.2. Four-fracture network. In this numerical experiment we want to see the performance of the proposed multigrid method when several fractures are considered. For this purpose, we consider a network of four fractures whose width is $d=10^{-2}$. We perform two different tests corresponding to nonconnected and connected fractures. In both cases, we consider a unit square porous medium domain with impermeable lateral walls and a given pressure on the top $(p=1)$ and the bottom $(p=0)$. The permeability of the porous matrix is given by the identity tensor, and the permeability in the fractures is given by $\mathbf{K}_{f i}=K_{f i} \mathbf{I}$, with $K_{f i}$ certain constants to be determined below, for $i=1,2,3,4$. Following $[8,31]$, immersed fracture tips are modeled by 


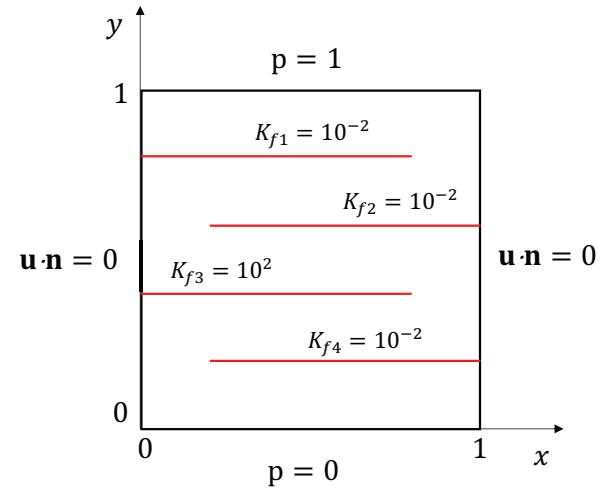

(a)

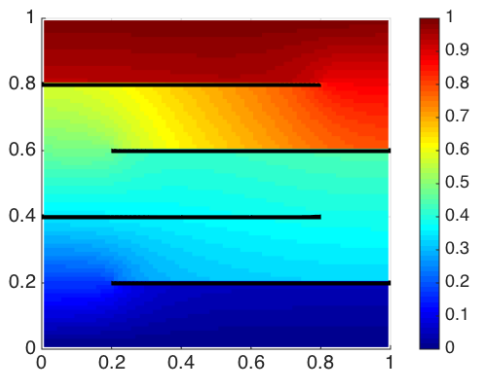

(b)

FIG. 12. (a) Fracture network and settings and (b) corresponding pressure distribution for the four-fracture experiment (Case 1).

homogeneous flux conditions. Note that, since we are using a reduced model for the fractures, locally refined grids are not required near the fracture tips.

4.2.1. Case 1: Four nonconnected fractures. The settings considered in this first case are based on [8] and schematized in Figure 12(a). A set of four horizontal fractures $\left\{\gamma_{i}\right\}_{i=1, \ldots, 4}$ is considered, where

$$
\begin{aligned}
& \gamma_{1}=\{(x, y) \mid y=0.8,0 \leq x \leq 0.8\} \\
& \gamma_{2}=\{(x, y) \mid y=0.6,0.2 \leq x \leq 1\} \\
& \gamma_{3}=\{(x, y) \mid y=0.4,0 \leq x \leq 0.8\} \\
& \gamma_{4}=\{(x, y) \mid y=0.2,0.2 \leq x \leq 1\}
\end{aligned}
$$

Fractures $\gamma_{1}, \gamma_{2}$, and $\gamma_{4}$ are assumed to be barriers with $K_{f 1}=K_{f 2}=K_{f 4}=10^{-2}$, whereas fracture $\gamma_{3}$ is highly conductive with $K_{f 3}=10^{2}$.

The effect of these fractures on the pressure distribution within the porous medium domain can be seen in Figure 12(b).

4.2.2. Case 2: Four connected fractures. In the second case, we assume a fracture network with four fractures which are connected. A schematic picture of the network together with the properties of the fractures can be seen in Figure 13(a).

In particular, we consider two vertical fractures

$$
\begin{aligned}
& \gamma_{3}=\{(x, y) \mid x=0.2,0 \leq y \leq 0.8\} \\
& \gamma_{4}=\{(x, y) \mid x=0.6,0 \leq y \leq 0.6\}
\end{aligned}
$$

with constant permeabilities given by $K_{f 3}=10^{2}$ and $K_{f 4}=10^{-2}$, respectively. Moreover, we consider two horizontal fractures

$$
\begin{aligned}
& \gamma_{1}=\{(x, y) \mid y=0.8,0 \leq x \leq 0.6\} \\
& \gamma_{2}=\{(x, y) \mid y=0.6,0.2 \leq x \leq 1\}
\end{aligned}
$$

The first one has a constant permeability given by $K_{f 1}=10^{2}$, whereas the second 


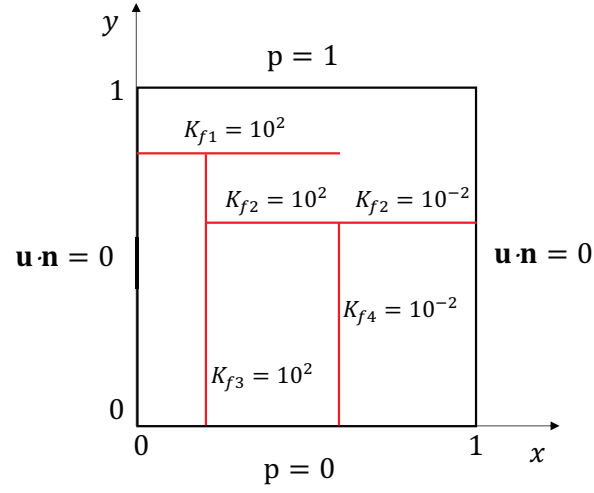

(a)

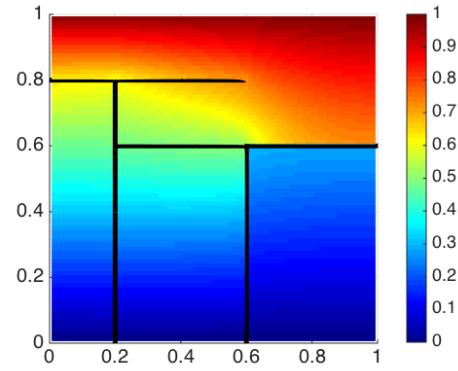

(b)

FIG. 13. (a) Fracture network and settings and (b) corresponding pressure distribution for the four-fracture experiment (Case 2).

TABLE 2

Number of iterations of the mixed-dimensional multigrid method necessary to solve the fourfracture experiments for different grid sizes.

\begin{tabular}{ccccccc} 
& $40 \times 40$ & $80 \times 80$ & $160 \times 160$ & $320 \times 320$ & $640 \times 640$ & $1280 \times 1280$ \\
\hline Case 1 & 9 & 9 & 10 & 10 & 11 & 11 \\
\hline \hline Case 2 & 11 & 11 & 11 & 12 & 13 & 13 \\
\hline
\end{tabular}

one has a variable permeability given by

$$
K_{f 2}= \begin{cases}10^{2}, & \text { if } 0.2 \leq x \leq 0.6 \\ 10^{-2}, & \text { if } 0.6<x \leq 1\end{cases}
$$

The pressure solution is shown in Figure 13(b), where we can observe the effect of the different permeabilities of the fractures on the pressure at the porous matrix.

We perform the proposed monolithic multigrid method for solving both test cases. Table 2 shows the number of multigrid iterations needed to reduce the initial residual in a factor of $10^{-10}$ for different mesh sizes. We can see a very robust behavior of the multigrid algorithm for both cases since few iterations are enough to satisfy the stopping criterion.

From this experiment, we observe that the monolithic mixed-dimensional multigrid method is also robust when several fractures (connected and/or nonconnected) are considered.

4.3. Benchmark problem. The last numerical experiment considered in this work is a benchmark problem for single-phase flow in fractured porous media stated in [28]. This test is based on a problem proposed in [36] with different boundary conditions and material properties. The fracture network embedded in the unit square domain is shown in Figure 14.

We have the Dirichlet condition $p=1$ on the right side. Homogeneous Neumann boundary conditions on the top and bottom are prescribed, whereas on the left side, we have a constant inflow flow, $\mathbf{u} \cdot \mathbf{n}=-1$ (see Figure 14). The permeability matrix is fixed to $\mathbf{K}=\mathbf{I}$, and all the fractures have a constant width $d=10^{-4}$. As in [28], we consider two cases for the permeability tensor in the fracture $\mathbf{K}_{f}=K_{f} \mathbf{I}$ : a case where 


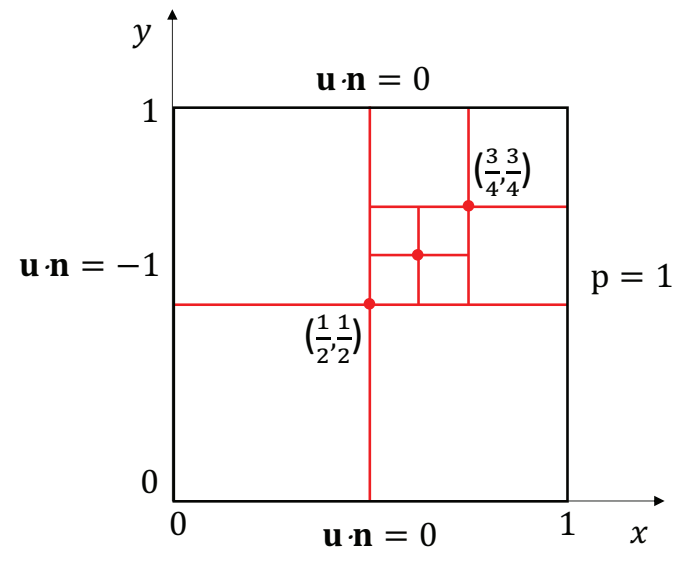

Fig. 14. Configuration and boundary conditions for the network of fractures in the benchmark problem.

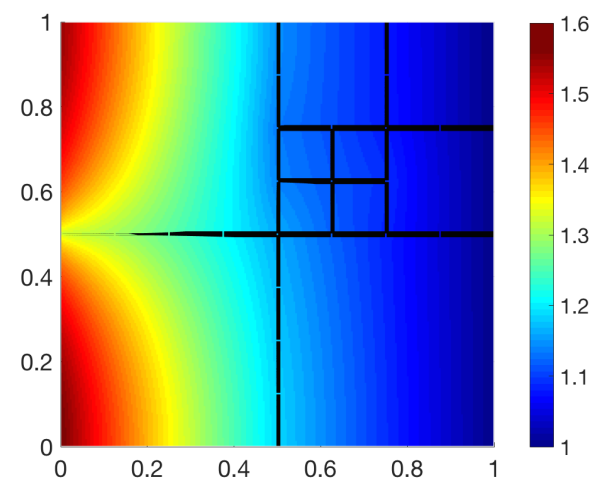

(a)

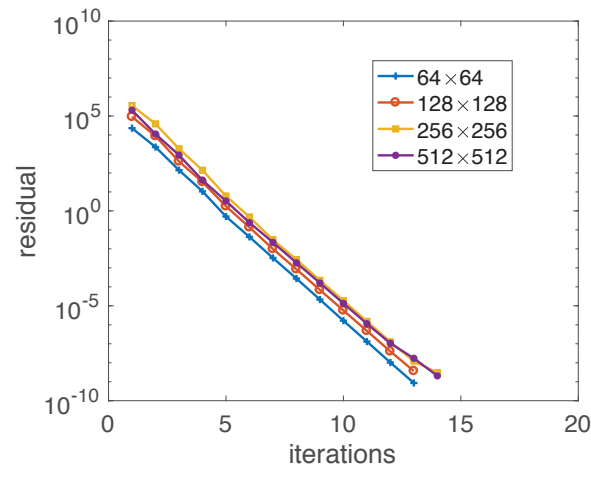

(b)

FIG. 15. (a) Pressure solution and (b) history of the convergence of the proposed multigrid method for the benchmark problem with highly conductive fractures.

the fluid tends to flow rapidly along the fracture, i.e., $K_{f}=10^{4}$, and a case where the fluid tends to avoid the fracture, i.e., $K_{f}=10^{-4}$. Both cases have been solved by the proposed mixed-dimensional multigrid method, giving rise to a very efficient solver independent of the fracture network.

For the first case in which we deal with a highly conductive network, the obtained pressure distribution is displayed in Figure 15(a). Such a distribution is in accordance with the reference solution of the benchmark problem published in [28].

The history of the convergence of the monolithic multigrid method is depicted in Figure 15(b), where the residual reduction is shown for different grid sizes. The stopping criterion is to reduce the initial residual until $10^{-8}$. We can observe that, as expected, the performance of the multigrid method is independent of the spatial discretization parameter. Moreover, it results in a very efficient solver since only around 10 iterations are enough to solve the whole fracture network.

Similar results are obtained for the second case, corresponding to a blocking fracture network. Again, the pressure distribution matches perfectly with the reference 


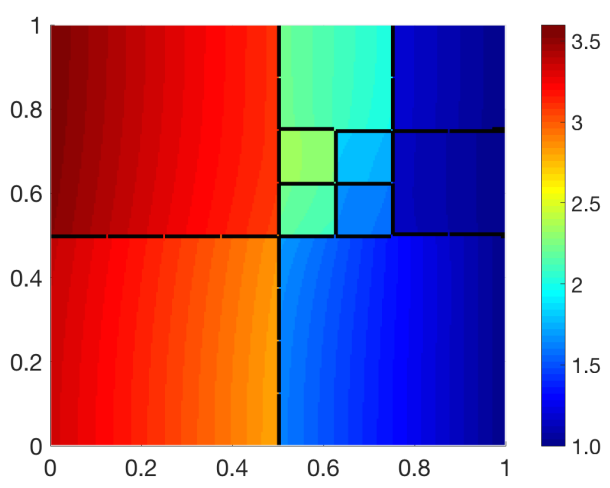

(a)

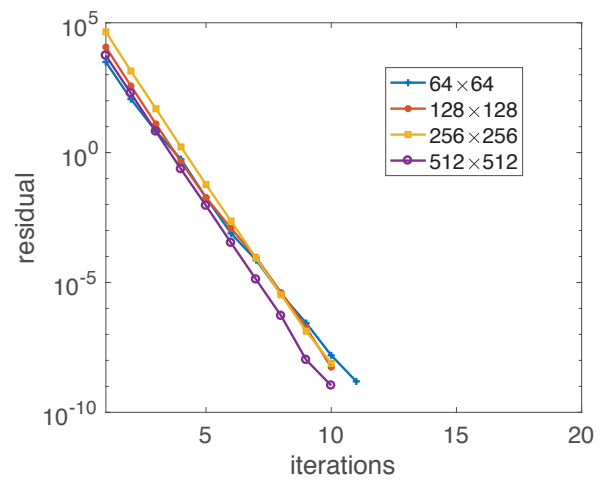

(b)

FIG. 16. (a) Pressure solution and (b) history of the convergence of the proposed multigrid method for the benchmark problem with blocking fractures.

solution in [28], as shown in Figure 16(a). We can observe in the picture the pressure discontinuities reminiscent of the low permeability in the fractures.

In Figure 16(b), we display the history of the convergence of the proposed mixeddimensional multigrid method for this second case of the benchmark problem. The convergence results for the blocking fracture network are similar to those presented for the conducting fractures. For both cases, the monolithic mixed-dimensional multigrid method shows an excellent performance.

4.4. Local Fourier analysis test. Local Fourier analysis (LFA) [17, 18, 81] is the main quantitative analysis to predict the convergence rates of multigrid algorithms. LFA assumes that all operators involved in the multigrid procedure are local, have constant coefficients, and are defined on an infinite grid neglecting the effect of boundary conditions. It seems not possible, or at least it is not clear, how to carry out LFA for the mixed-dimensional multigrid method proposed here. Nevertheless, we have performed LFA to predict the convergence of the multigrid based on the Vanka smoother for the Darcy problem considered in the porous matrix. The analysis of Vanka-type smoothers by LFA requires a special strategy, and here we have followed the ideas presented in [64]. A detailed explanation of the analysis performed here can be found in Appendix A. From this analysis, we observe that the convergence rates obtained for the whole problem including fractures are very close to those provided in the case of simple Darcy flow. This can be seen in Table 3, where the two-grid convergence factor predicted by LFA for Darcy flow is compared with the asymptotic convergence rates obtained in the numerical experiments carried out in this work. We consider here four smoothing steps, as in the numerical tests performed. It is observed that the computationally obtained convergence factors are very similar to the one estimated by LFA and vary from 0.04 in the first experiment to 0.085 in the benchmark problem, which represents the worst case. This means that the implementation and the treatment in the fractures is done in an optimal way since the convergence of the whole fracture network problem is very similar to that for the Darcy flow problem.

5. Conclusions. We have proposed a monolithic mixed-dimensional multigrid method for solving single-phase flow problems in porous media with intersecting fractures. The geometric multigrid algorithm is based on combining two-dimensional 
TABLE 3

Comparison between the predicted LFA two-grid convergence factor for the Darcy problem and the asymptotic convergence factors experimentally obtained from the numerical experiments performed in the fractured porous media.

\begin{tabular}{c|c|c|c|c|c}
\hline \multirow{2}{*}{ LFA } & \multicolumn{2}{|c|}{ One fracture } & \multicolumn{2}{c|}{ Four fractures } & \multirow{2}{*}{ Benchmark } \\
& Test 4.1.1 & Test 4.1.2 & Test 4.2.1 & Test 4.2.2 & \\
\hline \hline 0.04 & 0.039 & 0.077 & 0.045 & 0.08 & 0.085 \\
\hline
\end{tabular}

smoother and intergrid transfer operators in the porous matrix with their onedimensional counterparts within the fracture network. This exotic union gives rise to a very efficient solver for this type of problem. The robustness of the proposed method with respect to the permeability of the fractures, the number of fractures, and the grid size has been shown through different numerical experiments, including a benchmark problem from the literature.

A limitation of the proposed method is the need for a configuration of possibly intersecting horizontal and vertical fractures. The extension of the algorithm to more general situtations involving fractures with any orientation would require the use of unstructured coarse meshes. Accordingly, the discretization scheme, now based on the two-point flux approximation method, should be replaced by multipoint flux approximation techniques. This primary extension is under current research.

On the other hand, throughout this work, we have considered nondeformable porous media in the presence of fractures. A further extension of the proposed solver would be the approximation of flow problems in poroelastic fractured media. In the framework of hydraulic fracturing, such problems have been extensively tackled in the literature. Some significant contributions in this field can be found in $[20,22,27,37$, $42,46,53,55,58,60,61,68,70,71]$. More specifically, the crack propagation process has been handled using phase-field $[51,54]$ and XFEM $[74,75,76]$ methods. Some recent approaches to control the conditioning of these latter on elastic fractured media are proposed in $[1,2,3,4]$. The design of multigrid solvers for fracture problems in poroelastic media will be a topic of future research.

Appendix A. LFA for Darcy flow. In this appendix, we present the basis to perform LFA for a multigrid method for the Darcy equation based on the five-point Vanka smoother introduced in Figure 7(a) in section 3.3. To perform this analysis, we first extend the two-dimensional staggered grid considered in this work to an infinite mesh (see Figure 17).

In particular, this infinite grid is composed of three different types of grid points so that we can decompose the global mesh into three infinite subgrids as $G^{k}=G_{1}^{k} \cup$ $G_{2}^{k} \cup G_{3}^{k}$ such that

$$
G_{j}^{k}=\left\{\left(x_{j}, y_{j}\right)=\left(k_{1}, k_{2}\right) h_{k}+\left(\delta_{1}, \delta_{2}\right) \mid k_{1}, k_{2} \in \mathbb{Z}\right\},
$$

where

$$
\left(\delta_{1}, \delta 2\right)=\left\{\begin{array}{l}
(0,0) \text { for } G_{1}^{k} \text { pressure grid points, } \\
(h / 2,0) \text { for } G_{2}^{k} \text { grid points for the horizontal velocities } \\
(0, h / 2) \text { for } G_{3}^{k} \text { grid points for the vertical velocities. }
\end{array}\right.
$$

According to this decomposition of the infinite grid, we need to define suitable vectorgrid functions

$$
\varphi_{k}\left(\boldsymbol{\theta}, \mathbf{x}^{k}\right):=\left[\begin{array}{l}
e^{\imath \theta_{x} x_{1} / h_{k}} e^{\imath \theta_{y} y_{1} / h_{k}} \\
e^{\imath \theta_{x} x_{2} / h_{k}} e^{\imath \theta_{y} y_{2} / h_{k}} \\
e^{\imath \theta_{x} x_{3} / h_{k}} e^{\imath \theta_{y} y_{3} / h_{k}}
\end{array}\right]
$$




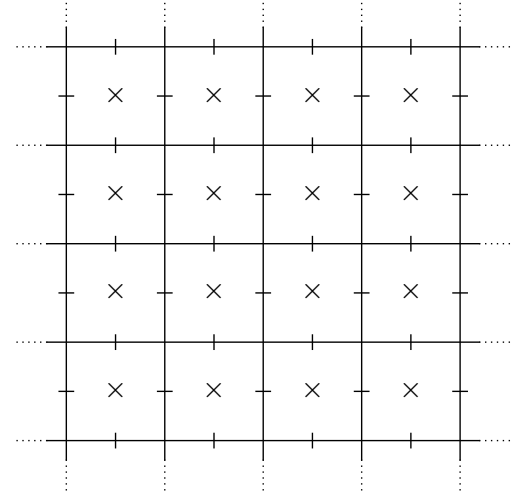

$\times$ pressure in the porous medium

+ velocity in the porous medium

FIG. 17. Infinite grid and location of the unknowns for the Darcy problem.

where $\boldsymbol{\theta}=\left(\theta_{x}, \theta_{y}\right), \mathbf{x}^{k}=\left(\mathbf{x}_{1}^{k}, \mathbf{x}_{2}^{k}, \mathbf{x}_{3}^{k}\right)$ with $\mathbf{x}_{j}^{k}=\left(x_{j}, y_{j}\right) \in G_{j}^{k}$, and $h_{k}$ is the spatial grid size in the mesh $G^{k}$, which is considered equal in every direction for simplicity in the presentation. These vector-grid functions are the so-called Fourier modes, and they form a unitary basis yielding the so-called Fourier space $\mathcal{F}\left(G^{k}\right):=$ $\operatorname{span}\left\{\varphi_{k}\left(\boldsymbol{\theta}, \mathbf{x}^{k}\right) ; \boldsymbol{\theta} \in \Theta=(-\pi, \pi]^{2}\right\}$. Notice that the definition of the Fourier components is different from the standard LFA on collocated grids and yields a more involved LFA approach. It is still fulfilled, however, that the Fourier modes are formal eigenfunctions of any discrete operator $A_{k}$ that satisfies the assumptions of the LFA stated in section 4.4 , that is, $A_{k} \varphi_{k}\left(\boldsymbol{\theta}, \mathbf{x}^{k}\right)=\widetilde{A}_{k}(\boldsymbol{\theta}) \varphi_{k}\left(\boldsymbol{\theta}, \mathbf{x}^{k}\right)$, where $\widetilde{A}_{k}(\boldsymbol{\theta})$ is the so-called Fourier symbol of operator $A_{k}$ and denotes the representation of such operator on the Fourier space. By denoting $u, v$, and $p$ the horizontal and vertical velocity and the pressure unknowns in our problem, respectively, we can write the discrete operator in block form as

$$
A_{k}=\left[\begin{array}{ccc}
A_{k}^{u u} & A_{k}^{u v} & A_{k}^{u p} \\
A_{k}^{v u} & A_{k}^{v v} & A_{k}^{v p} \\
A_{k}^{p u} & A_{k}^{p v} & A_{k}^{p p}
\end{array}\right]=\left[\begin{array}{ccc}
K^{-1} I & 0 & \partial_{x} \\
0 & K^{-1} I & \partial_{y} \\
\partial_{x} & \partial_{y} & 0
\end{array}\right]
$$

and the corresponding Fourier symbol would be given as

$$
\widetilde{A}_{k}=\left[\begin{array}{ccc}
\widetilde{A}_{k}^{u u} & \widetilde{A}_{k}^{u v} & \widetilde{A}_{k}^{u p} \\
\widetilde{A}_{k}^{v u} & \widetilde{A}_{k}^{v v} & \widetilde{A}_{k}^{v p} \\
\widetilde{A}_{k}^{p u} & \widetilde{A}_{k}^{p v} & \widetilde{A}_{k}^{p p}
\end{array}\right]=\left[\begin{array}{ccc}
K^{-1} & 0 & s_{x} \\
0 & K^{-1} & s_{y} \\
s_{x} & s_{y} & 0
\end{array}\right] \text {, where }\left\{\begin{array}{c}
s_{x}=\frac{2 \imath}{h} \sin \frac{\theta_{x}}{2}, \\
s_{y}=\frac{2 \imath}{h} \sin \frac{\theta_{y}}{2} .
\end{array}\right.
$$

Similar computations can be done to obtain the Fourier symbol of classical relaxation methods, which provides the relation between the initial and the fully corrected errors for the involved variables. Let us denote $e_{k}^{u, m}, e_{k}^{v, m}$, and $e_{k}^{p, m}$ the initial errors at $m$-iteration for $u, v$, and $p$, respectively, and $e_{k}^{u, m+1}, e_{k}^{v, m+1}$, and $e_{k}^{p, m+1}$ the corresponding final errors. The overlapping block smoothers considered here, however, require a special LFA. This is due to the fact that they update some variable more than once because of the overlapping of the local subdomains which are simultaneously solved, and this has to be taken into account in the analysis since it causes some intermediate errors to appear in addition to the initial and final errors. Since the velocities are the unknowns that are updated twice, we need to introduce the intermediate errors $e_{k}^{u, m+\frac{1}{2}}$ and $e_{k}^{v, m+\frac{1}{2}}$. Following the LFA techniques presented in 
$[50,57,64,72]$, we apply this analysis to the five-point Vanka smother considered for the two-dimensional Darcy flow. This smoother simultaneously updates the equations of the unknowns $p_{i, j}, u_{i+1 / 2, j}, u_{i-1 / 2, j}, v_{i, j+1 / 2}$, and $v_{i, j-1 / 2}$, as shown in Figure 7(a). We can write the system to solve for each pressure grid point $(i, j)$ in terms of corrections and residuals as follows:

$$
\left[\begin{array}{ccccc}
K^{-1} & 0 & 0 & 0 & -\frac{1}{h} \\
0 & K^{-1} & 0 & 0 & -\frac{1}{h} \\
0 & 0 & K^{-1} & 0 & \frac{1}{h} \\
0 & 0 & 0 & K^{-1} & \frac{1}{h} \\
\frac{1}{h} & \frac{1}{h} & -\frac{1}{h} & -\frac{1}{h} & 0
\end{array}\right]\left[\begin{array}{c}
\delta u_{i+1 / 2, j} \\
\delta v_{i, j+1 / 2} \\
\delta u_{i-1 / 2, j} \\
\delta v_{i, j-1 / 2} \\
\delta p_{i, j}
\end{array}\right]=\left[\begin{array}{c}
r_{i+1 / 2, j}^{u} \\
r_{i, j+1 / 2}^{v} \\
r_{i-1 / 2, j}^{u} \\
r_{i, j-1 / 2}^{v} \\
r_{i, j}^{p}
\end{array}\right]
$$

Taking into account that within such a block it is the first time that we update $u_{i+1 / 2, j}$ and $v_{i, j+1 / 2}$, whereas $u_{i-1 / 2, j}$ and $v_{i, j-1 / 2}$ are updated for the second time, we can write the corrections in terms of the errors as follows:

$$
\begin{aligned}
\delta u_{i+1 / 2, j} & =e_{k}^{u, m+\frac{1}{2}}\left(x_{i+1 / 2, j}^{2}\right)-e_{k}^{u, m}\left(x_{i+1 / 2, j}^{2}\right), \\
\delta v_{i, j+1 / 2} & =e_{k}^{v, m+\frac{1}{2}}\left(x_{i, j+1 / 2}^{3}\right)-e_{k}^{v, m}\left(x_{i, j+1 / 2}^{3}\right), \\
\delta u_{i-1 / 2, j} & =e_{k}^{u, m+1}\left(x_{i-1 / 2, j}^{2}\right)-e_{k}^{u, m+\frac{1}{2}}\left(x_{i-1 / 2, j}^{2}\right), \\
\delta v_{i, j-1 / 2} & =e_{k}^{v, m+1}\left(x_{i, j-1 / 2}^{3}\right)-e_{k}^{v, m+\frac{1}{2}}\left(x_{i, j-1 / 2}^{3}\right), \\
\delta p_{i, j} & =e_{k}^{p, m+1}\left(x_{i, j}^{1}\right)-e_{k}^{p, m}\left(x_{i, j}^{1}\right) .
\end{aligned}
$$

Similarly, we can write the residuals on the right-hand side in terms of the errors. The LFA assumptions state that the errors can be written as a formal linear combination of Fourier modes. Without loss of generality, let the initial, intermediate, and fully corrected errors be given by a single Fourier mode multiplied by a coefficient $\alpha_{u}^{(l)}(\boldsymbol{\theta})$, $\alpha_{v}^{(l)}(\boldsymbol{\theta})$, or $\alpha_{p}^{(l)}(\boldsymbol{\theta})$, where the superscript $l$ represents the number of times that the variable in the subscript has been already updated in the current smoothing iteration $(l=0,1,2)$. In this way, (A.1) can be rewritten in terms of such coefficients as

$$
P\left[\begin{array}{c}
\alpha_{u}^{(1)}(\boldsymbol{\theta}) \\
\alpha_{v}^{(1)}(\boldsymbol{\theta}) \\
\alpha_{u}^{(2)}(\boldsymbol{\theta}) \\
\alpha_{v}^{(2)}(\boldsymbol{\theta}) \\
\alpha_{p}^{(1)}(\boldsymbol{\theta})
\end{array}\right]=Q\left[\begin{array}{c}
\alpha_{u}^{(0)}(\boldsymbol{\theta}) \\
\alpha_{v}^{(0)}(\boldsymbol{\theta}) \\
\alpha_{p}^{(0)}(\boldsymbol{\theta})
\end{array}\right]
$$

so that the updated Fourier coefficients can be given in terms of the nonupdated ones as follows:

$$
\left[\begin{array}{c}
\alpha_{u}^{(1)}(\boldsymbol{\theta}) \\
\alpha_{v}^{(1)}(\boldsymbol{\theta}) \\
\alpha_{u}^{(2)}(\boldsymbol{\theta}) \\
\alpha_{v}^{(2)}(\boldsymbol{\theta}) \\
\alpha_{p}^{(1)}(\boldsymbol{\theta})
\end{array}\right]=\left(P^{-1} Q\right)\left[\begin{array}{c}
\alpha_{u}^{(0)}(\boldsymbol{\theta}) \\
\alpha_{v}^{(0)}(\boldsymbol{\theta}) \\
\alpha_{p}^{(0)}(\boldsymbol{\theta})
\end{array}\right]
$$

From the previous expression, we can obtain the relation between the initial and fully corrected errors, which is the Fourier symbol of the relaxation, by considering the 
bottom $3 \times 3$ block of matrix $\left(P^{-1} Q\right)$, that is,

$$
\left[\begin{array}{l}
\alpha_{u}^{(2)}(\boldsymbol{\theta}) \\
\alpha_{v}^{(2)}(\boldsymbol{\theta}) \\
\alpha_{p}^{(1)}(\boldsymbol{\theta})
\end{array}\right]=\widetilde{S}_{k}(\boldsymbol{\theta})\left[\begin{array}{c}
\alpha_{u}^{(0)}(\boldsymbol{\theta}) \\
\alpha_{v}^{(0)}(\boldsymbol{\theta}) \\
\alpha_{p}^{(0)}(\boldsymbol{\theta})
\end{array}\right] \text {, where } \widetilde{S}_{k}(\boldsymbol{\theta})=\left(P^{-1} Q\right)_{\{3: 5,1: 3\}}
$$

In order to study the behavior of the two-grid cycle (and as a consequence of the multigrid method), we investigate the effect of the multigrid components and consequently of the two-grid operator $M_{k, k-1}=S_{k}^{\nu_{1}}\left(I_{h}-P_{k-1}^{k} A_{k-1}^{-1} R_{k}^{k-1} A_{k}\right) S_{k}^{\nu_{2}}$ acting on the Fourier space. With this purpose, we need to distinguish the low- and highfrequency Fourier components. This distinction depends on the coarsening strategy under consideration. Since standard coarsening (i.e., coarsening by a factor of two) is assumed here, the low- and high-frequency sets are given by $\Theta_{\text {low }}=\left(-\frac{\pi}{2}, \frac{\pi}{2}\right]^{2}$ and $\Theta_{\text {high }}=\Theta \backslash \Theta_{\text {low }}$, respectively. This is important because in the transition from the fine grid $G^{k}$ to the coarse grid $G^{k-1}$, each low frequency $\boldsymbol{\theta}=\boldsymbol{\theta}^{00} \in \Theta_{\text {low }}$ is coupled with three high frequencies, $\boldsymbol{\theta}^{11}, \boldsymbol{\theta}^{10}$, and $\boldsymbol{\theta}^{01}$, given by

$$
\boldsymbol{\theta}^{i j}=\boldsymbol{\theta}^{00}-\left(i \operatorname{sign}\left(\theta_{x}\right), j \operatorname{sign}\left(\theta_{y}\right)\right) \pi, i, j=0,1 .
$$

This coupling gives rise to a decomposition of the Fourier space into four-dimensional subspaces, the so-called subspaces of $2 h$-harmonics, which are generated by the Fourier modes associated with coupled frequencies as follows:

$$
\mathcal{F}^{4}\left(\boldsymbol{\theta}^{00}\right):=\operatorname{span}\left\{\varphi_{k}\left(\boldsymbol{\theta}^{00}, \cdot\right), \varphi_{k}\left(\boldsymbol{\theta}^{11}, \cdot\right), \varphi_{k}\left(\boldsymbol{\theta}^{10}, \cdot\right), \varphi_{k}\left(\boldsymbol{\theta}^{01}, \cdot\right)\right\}, \text { where } \boldsymbol{\theta}^{00} \in \Theta_{\text {low }} .
$$

Since the two-grid operator leaves the four-dimensional subspaces $\mathcal{F}^{4}\left(\boldsymbol{\theta}^{00}\right)$ invariant, the representation of $M_{k, k-1}$ on the Fourier space, $\widetilde{M}_{k, k-1}(\boldsymbol{\theta})$, has a block-diagonal structure regarding the partitioning in $2 h$-harmonics, and therefore it is possible to efficiently calculate the LFA two-grid convergence factor $\rho_{2 g}=\rho\left(M_{k, k-1}\right)$ as the maximum of the spectral radius of the corresponding $4 \times 4$ blocks, that is,

$$
\rho_{2 g}=\sup _{\boldsymbol{\theta}^{\mathrm{o0} \in \Theta_{l o w}}} \rho\left(\widetilde{M}_{k, k-1}\left(\boldsymbol{\theta}^{00}\right)\right) .
$$

\section{REFERENCES}

[1] K. Agathos, S. P. A. Bordas, and E. Chatzi, Improving the conditioning of XFEM/GFEM for fracture mechanics problems through enrichment quasi-orthogonalization, Comput. Methods Appl. Mech. Engrg., 346 (2019), pp. 1051-1073.

[2] K. Agathos, E. Chatzi, and S. P. A. Bordas, Stable 3D extended finite elements with higher order enrichment for accurate non planar fracture, Comput. Methods Appl. Mech. Engrg., 306 (2016), pp. 19-46.

[3] K. Agathos, E. Chatzi, S. P. A. Bordas, and D. Talaslidis, A well-conditioned and optimally convergent XFEM for $3 D$ linear elastic fracture, Internat. J. Numer. Methods Engrg., 105 (2016), pp. 643-677.

[4] K. Agathos, G. Ventura, E. Chatzi, and S. P. A. Bordas, Stable 3D XFEM/vector level sets for non-planar $3 D$ crack propagation and comparison of enrichment schemes, Internat. J. Numer. Methods Engrg., 113 (2018), pp. 252-276.

[5] R. Ahmed, M. G. Edwards, S. Lamine, B. A. H. Huisman, and M. Pal, Control-volume distributed multi-point flux approximation coupled with a lower-dimensional fracture model, J. Comput. Phys., 284 (2015), pp. 462-489.

[6] C. Alboin, J. Jaffré, J. E. Roberts, and C. Serres, Modeling fractures as interfaces for flow and transport in porous media, in Fluid Flow and Transport in Porous Media: Mathematical and Numerical Treatment, Contemp. Math. 295, American Mathematical Society, Providence, RI, 2002, pp. 13-24. 
[7] L. Amir, M. Kern, J. E. Roberts, And V. Martin, Décomposition de domaine pour un milieu poreux fracturé: Un modèle en $3 D$ avec fractures qui s'intersectent, ARIMA, 5 (2006), pp. 11-25.

[8] P. Angot, F. Boyer, And F. Hubert, Asymptotic and numerical modelling of flows in fractured porous media, ESAIM Math. Model. Numer. Anal., 43 (2009), pp. 239-275.

[9] P. F. Antonietti, C. Facciolà, A. Russo, and M. Verani, Discontinuous Galerkin Approximation of Flows in Fractured Porous Media on Polytopic Grids, Technical report MOX-55/2016, Dipartimento di Matematica, Politecnico di Milano, Milano, Italy, 2016.

[10] P. F. Antonietti, L. Formaggia, A. Scotti, M. Verani, and N. Verzott, Mimetic finite difference approximation of flows in fractured porous media, ESAIM Math. Model. Numer. Anal., 50 (2016), pp. 809-832.

[11] T. Arbogast and J. Douglas, JR., Dual-porosity models for flow in naturally fractured reservoirs, in Dynamics of Fluids in Hierarchical Porous Media, J. Cushman, ed., Academic Press, London, 1990, pp. 177-221.

[12] T. Arbogast, J. Douglas, Jr., and U. Hornung, Derivation of the double porosity model of single phase flow via homogenization theory, SIAM J. Math. Anal., 21 (1990), pp. 823-836.

[13] T. Arbogast, M. F. Wheeler, And I. Yotov, Mixed finite elements for elliptic problems with tensor coefficients as cell-centered finite differences, SIAM J. Numer. Anal., 34 (1997), pp. 828-852.

[14] M. F. Benedetto, S. Berrone, S. Pieraccini, and S. Scialò, The virtual element method for discrete fracture network simulations, Comput. Methods Appl. Mech. Engrg., 280 (2014), pp. $135-156$.

[15] W. M. Boon, J. M. Nordbotten, and J. E. Vatne, Mixed-Dimensional Elliptic Partial Differential Equations, preprint, arXiv:1710.00556 [math.AP], 2017.

[16] W. M. Boon, J. M. Nordbotten, and I. Yotov, Robust discretization of flow in fractured porous media, SIAM J. Numer. Anal., 56 (2018), pp. 2203-2233.

[17] A. Brandt, Multi-level adaptive solutions to boundary-value problems, Math. Comp., 31 (1977), pp. 333-390.

[18] A. BRAndt, Rigorous quantitative analysis of multigrid. I. Constant coefficients two-level cycle with $L_{2}$-norm, SIAM J. Numer. Anal., 31 (1994), pp. 1695-1730.

[19] M. Bukač, I. Yotov, And P. Zunino, Dimensional model reduction for flow through fractures in poroelastic media, ESAIM Math. Model. Numer. Anal., 51 (2017), pp. 1429-1471.

[20] T. D. CaO, F. Hussain, And B. A. Schrefler, Porous media fracturing dynamics: Stepwise crack advancement and fluid pressure oscillations, J. Mech. Phys. Solids, 111 (2018), pp. 113-133.

[21] F. Chave, D. A. Di Pietro, and L. Formaggia, A hybrid high-order method for Darcy flows in fractured porous media, SIAM J. Sci. Comput., 40 (2018), pp. A1063-A1094.

[22] Y. Chen, H. Lian, W. Liang, J. Yang, V. P. Nguyen, and S. P. A. Bordas, The influence of fracture geometry variation on non-Darcy flow in fractures under confining stresses, Int. J. Rock Mech. Min. Sci., 113 (2019), pp. 59-71.

[23] C. D'Angelo and A. Scotti, A mixed finite element method for Darcy flow in fractured porous media with non-matching grids, ESAIM Math. Model. Numer. Anal., 46 (2012), pp. $465-489$.

[24] J.-R. de Dreuzy, G. Pichot, B. Poirriez, And J. Erhel, Synthetic benchmark for modeling flow in 3D fractured media, Comput. Geosci., 50 (2013), pp. 59-71.

[25] M. Del Pra, A. Fumagalli, And A. Scotti, Well posedness of fully coupled fracture/bulk Darcy flow with XFEM, SIAM J. Numer. Anal., 55 (2017), pp. 785-811.

[26] R. Eymard, T. Gallouët, C. Guichard, R. Herbin, and R. Masson, TP or not TP, that is the question, Comput. Geosci., 18 (2014), pp. 285-296.

[27] Y. FENG AND K. E. GRAY, XFEM-based cohesive zone approach for modeling near-wellbore hydraulic fracture complexity, Acta Geotech., 14 (2019), pp. 377-402.

[28] B. Flemisch, I. Berre, W. Boon, A. Fumagalli, N. Schwenck, A. Scotti, I. Stefansson, AND A. TATOMIR, Benchmarks for single-phase flow in fractured porous media, Adv. Water Resour., 111 (2018), pp. 239-258.

[29] B. Flemisch, A. Fumagalli, and A. Scotti, A review of the XFEM-based approximation of flow in fractured porous media, in Advances in Discretization Methods, SEMA SIMAI Springer Ser. 12, Springer, Cham, Switzerland, 2016, pp. 47-76.

[30] L. Formaggia, A. Fumagalli, A. Scotti, and P. Ruffo, A reduced model for Darcy's problem in networks of fractures, ESAIM Math. Model. Numer. Anal., 48 (2014), pp. 1089-1116.

[31] L. Formaggia, A. Scotti, and F. Sottocasa, Analysis of a mimetic finite difference approximation of flows in fractured porous media, ESAIM Math. Model. Numer. Anal., 52 (2018), pp. 595-630. 
[32] N. Frih, V. Martin, J. E. Roberts, And A. SaÂda, Modeling fractures as interfaces with nonmatching grids, Comput. Geosci., 16 (2012), pp. 1043-1060.

[33] N. Frih, J. E. Roberts, AND A. SAAdA, Modeling fractures as interfaces: A model for Forchheimer fractures, Comput. Geosci., 12 (2008), pp. 91-104.

[34] A. Fumagalli and E. Keilegavlen, Dual virtual element method for discrete fractures networks, SIAM J. Sci. Comput., 40 (2018), pp. B228-B258.

[35] A. Fumagalli And A. Scotti, A numerical method for two-phase flow in fractured porous media with non-matching grids, Adv. Water Resour., 62 (2013), pp. 454-464.

[36] S. Geiger, M. Dentz, And I. Neuweiler, A novel multi-rate dual-porosity model for improved simulation of fractured and multi-porosity reservoirs, SPE J., 18 (2013), pp. 670-684.

[37] V. Girault, K. Kumar, And M. F. WheEler, Convergence of iterative coupling of geomechanics with flow in a fractured poroelastic medium, Comput. Geosci., 20 (2016), pp. 997-1011.

[38] V. Girault, M. F. Wheeler, B. Ganis, and M. E. Mear, A lubrication fracture model in a poro-elastic medium, Math. Models Methods Appl. Sci., 25 (2015), pp. 587-645.

[39] D. Gläser, R. Helmig, B. Flemisch, and H. Class, A discrete fracture model for two-phase flow in fractured porous media, Adv. Water Resour., 110 (2017), pp. 335-348.

[40] W. Hackbusch, Multi-Grid Methods and Applications, Springer, Berlin, 1985.

[41] H. Hajibeygi, D. Karvounis, and P. Jenny, A hierarchical fracture model for the iterative multiscale finite volume method, J. Comput. Phys., 230 (2011), pp. 8729-8743.

[42] K. K. Hanowski And O. Sander, Simulation of Deformation and Flow in Fractured, Poroelastic Materials, preprint, arXiv:1606.05765 [math.NA], 2016.

[43] T.-T.-P. Hoang, C. Japhet, M. Kern, and J. E. Roberts, Space-time domain decomposition for reduced fracture models in mixed formulation, SIAM J. Numer. Anal., 54 (2016), pp. 288-316.

[44] E. Keilegavlen, A. Fumagaldi, R. Berge, and I. Stefansson, Implementation of MixedDimensional Models for Flow in Fractured Porous Media, preprint, arXiv:1712.07392 [Cs.CE], 2017.

[45] P. KNABner And J. E. RoBerts, Mathematical analysis of a discrete fracture model coupling Darcy flow in the matrix with Darcy-Forchheimer flow in the fracture, ESAIM Math. Model. Numer. Anal., 48 (2014), pp. 1451-1472.

[46] B. Lecampion, An extended finite element method for hydraulic fracture problems, Commun. Numer. Methods Engrg., 25 (2009), pp. 121-133.

[47] M. Lesinigo, C. D'Angelo, And A. Quarteroni, A multiscale Darcy-Brinkman model for fluid flow in fractured porous media, Numer. Math., 117 (2011), pp. 717-752.

[48] P. Luo, C. Rodrigo, F. J. Gaspar, and C. W. Oosterlee, Uzawa smoother in multigrid for the coupled porous medium and Stokes flow system, SIAM J. Sci. Comp., 39 (2017), pp. S633-S661.

[49] P. Luo, C. Rodrigo, F. J. Gaspar, and C. W. Oosterlee, Monolithic multigrid method for the coupled Stokes flow and deformable porous medium system, J. Comput. Phys., 353 (2018), pp. 148-168.

[50] S. P. MacLachlan and C. W. Oosterlee, Local Fourier analysis for multigrid with overlapping smoothers applied to systems of PDEs, Numer. Linear Algebra Appl., 18 (2011), pp. $751-774$.

[51] T. K. Mandal, V. P. NGuyen, and A. Heidarpour, Phase field and gradient enhanced damage models for quasi-brittle failure: A numerical comparative study, Eng. Fract. Mech., 207 (2019), pp. 48-67.

[52] V. Martin, J. Jaffré, And J. E. Roberts, Modeling fractures and barriers as interfaces for flow in porous media, SIAM J. Sci. Comp., 26 (2005), pp. 1667-1691.

[53] E. Mikaeili ANd B. SChrefler, XFEM, strong discontinuities and second-order work in shear band modeling of saturated porous media, Acta Geotech., 13 (2018), pp. 1249-1264.

[54] A. Mikelić, M. F. Wheeler, AND T. Wick, Phase-field modeling of a fluid-driven fracture in a poroelastic medium, Comput. Geosci., 19 (2015), pp. 1171-1195.

[55] E. Milanese, T. D. CaO, L. Simoni, and B. A. Schrefler, Fracturing in dry and saturated porous media, in Advances in Computational Plasticity, E. Oñate, D. Peric, E. de Souza Neto, and M. Chiumenti, eds., Comput. Methods Appl. Sci. 46, Springer, Dordrecht, The Netherlands, 2018, pp. 265-288.

[56] A. Moinfar, A. Varavei, K. Sepehrnoori, and R. T. Johns, Development of an efficient embedded discrete fracture model for $3 D$ compositional reservoir simulation in fractured reservoirs, SPE J., 19 (2014), pp. 289-303.

[57] J. MolenaAr, A two-grid analysis of the combination of mixed finite elements and Vanka-type relaxation, in Multigrid Methods III, W. Hackbusch and U. Trottenberg, eds., Internat. Ser. Numer. Math. 98, Birkhäuser, Basel, Switzerland, 1991, pp. 313-324. 
[58] V. P. Nguyen, H. Lian, T. Rabczuk, And S. Bordas, Modelling hydraulic fractures in porous media using flow cohesive interface elements, Eng. Geol., 225 (2017), pp. 68-82.

[59] J. M. Nordbotten, W. M. Boon, A. Fumagalli, and E. Keilegavlen, Unified Approach to Discretization of Flow in Fractured Porous Media, preprint, arXiv:1802.05961 [math.NA], 2018.

[60] A. Paluszny, S. Salimzadeh, and R. W. Zimmerman, Finite-element modeling of the growth and interaction of hydraulic fractures in poroelastic rock formations, in Hydraulic Fracture Modeling, Y.-S. Wu, ed., Elsevier, Oxford, 2018, pp. 1-19.

[61] F. Pesavento, B. A. Schrefler, and G. Sciumè, Multiphase flow in deforming porous media: A review, Arch. Comput. Methods Eng., 24 (2017), pp. 423-448.

[62] C. Rodrigo, Geometric Multigrid Methods on Triangular Grids. Application to SemiStructured Meshes, Lambert Academic Publishing, Riga, Latvia, 2012.

[63] C. Rodrigo, F. J. Gaspar, and F. J. Lisbona, Multigrid methods on semi-structured grids, Arch. Comput. Methods Engrg., 19 (2012), pp. 499-538.

[64] C. Rodrigo, F. J. Gaspar, and F. J. Lisbona, On a local Fourier analysis for overlapping block smoothers on triangular grids, Appl. Numer. Math., 105 (2016), pp. 96-111.

[65] T. F. Russell And M. F. WheEler, Finite element and finite difference methods for continuous flows in porous media, in The Mathematics of Reservoir Simulation, R. E. Ewing, ed., Frontiers in Applied Mathematics 1, SIAM, Philadelphia, 1983, pp. 35-106.

[66] T. H. Sandve, I. Berre, And J. M. Nordbotten, An efficient multi-point flux approximation method for discrete fracture-matrix simulations, J. Comput. Phys., 231 (2012), pp. 37843800.

[67] T. H. Sandve, E. Keilegavlen, and J. M. Nordbotten, Physics-based preconditioners for flow in fractured porous media, Water Resour. Res., 50 (2014), pp. 1357-1373.

[68] B. A. Schrefler And L. Simoni, A unified approach to the analysis of saturated-unsaturated elastoplastic porous media, in Numerical Methods in Geomechanics, Vol. 1, Balkema, Rotterdam, The Netherlands, 1988, pp. 205-212.

[69] N. Schwenck, B. Flemisch, R. Helmig, and B. I. Wohlmuth, Dimensionally reduced flow models in fractured porous media: Crossings and boundaries, Comput. Geosci., 19 (2015), pp. 1219-1230.

[70] M. Sheng, G. Li, D. Sutula, S. Tian, and S. P. A. Bordas, XFEM modeling of multistage hydraulic fracturing in anisotropic shale formations, J. Petrol. Sci. Engrg., 162 (2018), pp. 801-812.

[71] L. Simoni And B. A. Schrefler, Multi field simulation of fracture, in Advances in Applied Mechanics, Vol. 47, S. P. A. Bordas, ed., Academic Press, London, 2014, pp. 367-519.

[72] S. Sivaloganathan, The use of local mode analysis in the design and comparison of multigrid methods, Comput. Phys. Commun., 65 (1991), pp. 246-252.

[73] K. Stüben and U. Trottenberg, Multigrid methods: Fundamental algorithms, model problem analysis and applications, in Multigrid Methods, W. Hackbusch and U. Trottenberg, eds., Lecture Notes in Math. 960, Springer, Berlin, 1982, pp. 1-176.

[74] D. Sutula, P. Kerfriden, T. van Dam, and S. P. A. Bordas, Minimum energy multiple crack propagation. Part I: Theory and state of the art review, Eng. Fract. Mech., 191 (2018), pp. 205-224.

[75] D. Sutula, P. Kerfriden, T. van Dam, and S. P. A. Bordas, Minimum energy multiple crack propagation. Part-II: Discrete solution with XFEM, Eng. Fract. Mech., 191 (2018), pp. 225-256.

[76] D. Sutula, P. Kerfriden, T. van Dam, and S. P. A. Bordas, Minimum energy multiple crack propagation. Part III: XFEM computer implementation and applications, Eng. Fract. Mech., 191 (2018), pp. 257-276.

[77] M. Tene, M. S. A. Kobaisi, And H. Hajibeygi, Algebraic multiscale method for flow in heterogeneous porous media with embedded discrete fractures (F-AMS), J. Comput. Phys., 321 (2016), pp. 819-845.

[78] U. Trottenberg, C. W. Oosterlee, and A. Schüller, Multigrid, Academic Press, New York, 2001.

[79] S. P. VANKA, Block-implicit multigrid solution of Navier-Stokes equations in primitive variables, J. Comput. Phys., 65 (1986), pp. 138-158.

[80] P. Wesseling, An Introduction to Multigrid Methods, John Wiley \& Sons, Chichester, UK, 1992.

[81] R. Wienands and W. Joppich, Practical Fourier Analysis for Multigrid Methods, Chapman \& Hall/CRC Press, Boca Raton, FL, 2005. 